OPEN ACCESS

Edited by:

Weiwen Zhang,

Tianjin University, China

Reviewed by:

Yinhua Lu,

Institute of Plant Physiology and

Ecology - SIBS (CAS), China

Junbiao Dai,

Tsinghua University, China

${ }^{*}$ Correspondence:

Asunción Contreras contrera@ua.es

Specialty section:

This article was submitted to Microbial Physiology and Metabolism, a section of the journal Frontiers in Microbiology

Received: 11 May 2017 Accepted: 20 June 2017

Published: 11 July 2017

Citation:

Labella Jl, Cantos R, Espinosa J,

Forcada-Nadal A, Rubio $V$ and Contreras A (2017) PipY, a Member of the Conserved COG0325 Family of PLP-Binding Proteins, Expands the Cyanobacterial Nitrogen Regulatory Network.

Front. Microbiol. 8:1244. doi: 10.3389/fmicb.2017.01244

\section{PipY, a Member of the Conserved COG0325 Family of PLP-Binding Proteins, Expands the Cyanobacterial Nitrogen Regulatory Network}

\author{
José I. Labella ${ }^{1}$, Raquel Cantos ${ }^{1}$, Javier Espinosa ${ }^{1}$, Alicia Forcada-Nadal ${ }^{1,2}$, \\ Vicente Rubio ${ }^{2,3}$ and Asunción Contreras ${ }^{1 *}$
}

\begin{abstract}
'Departamento de Fisiología, Genética y Microbiología, Universidad de Alicante, Alicante, Spain, ${ }^{2}$ Instituto de Biomedicina de Valencia - Consejo Superior de Investigaciones Científicas, Valencia, Spain, ${ }^{3}$ Group 739, Centro de Investigación Biomédica en Red de Enfermedades Raras - Instituto de Salud Carlos III, Valencia, Spain
\end{abstract}

Synechococcus elongatus PCC 7942 is a paradigmatic model organism for nitrogen regulation in cyanobacteria. Expression of genes involved in nitrogen assimilation is positively regulated by the 2-oxoglutarate receptor and global transcriptional regulator NtcA. Maximal activation requires the subsequent binding of the co-activator PipX. PII, a protein found in all three domains of life as an integrator of signals of the nitrogen and carbon balance, binds to PipX to counteract NtcA activity at low 2-oxoglutarate levels. PII-PipX complexes can also bind to the transcriptional regulator PImA, whose regulon remains unknown. Here we expand the nitrogen regulatory network to PipY, encoded by the bicistronic operon pipXY in S. elongatus. Work with PipY, the cyanobacterial member of the widespread family of COG0325 proteins, confirms the conserved roles in vitamin B6 and amino/keto acid homeostasis and reveals new PLP-related phenotypes, including sensitivity to antibiotics targeting essential PLP-holoenzymes or synthetic lethality with cysK. In addition, the related phenotypes of pipY and pipX mutants are consistent with genetic interactions in the contexts of survival to PLP-targeting antibiotics and transcriptional regulation. We also showed that PipY overexpression increased the length of $S$. elongatus cells. Taken together, our results support a universal regulatory role for COG0325 proteins, paving the way to a better understanding of these proteins and of their connections with other biological processes.

Keywords: cyanobacteria, PipX, COG0325, PipY, nitrogen regulation

\section{INTRODUCTION}

Cyanobacteria are phototrophic organisms that perform oxygenic photosynthesis and require the assimilation of ammonia for autotrophic growth. This assimilation is carried out by the GS-GOGAT cycle, consuming 2-oxoglutarate (2-OG) (Muro-Pastor et al., 2001, 2005) which is a master regulator metabolite at the intersection between the carbon and nitrogen metabolic pathways, an thus an excellent indicator of the carbon to nitrogen balance (Muro-Pastor et al., 2001; Forchhammer, 2004; Laurent et al., 2005; Huergo and Dixon, 2015). 2-OG modulates the 
interactions of three key cyanobacterial proteins, two of which, the signal transducer PII and the global transcriptional regulator NtcA, bind 2-OG. The PipX protein can form alternative complexes with NtcA and PII and these interactions are, respectively, stimulated and inhibited by $2-\mathrm{OG}$, providing a mechanistic link between PII signaling and NtcA-regulated gene expression (Espinosa et al., 2006). PipX binds to either, 2OG-bound NtcA to stimulate DNA binding and transcriptional activity, or to 2-OG-free PII to form PII-PipX complexes (Tanigawa et al., 2002; Vazquez-Bermudez et al., 2002; Llacer et al., 2010; Zhao et al., 2010; Forcada-Nadal et al., 2014), which in turn can bind to the transcriptional regulator PlmA (Labella et al., 2016). While PII is found in all three domains of life as integrator of signals of the nitrogen and carbon balance, PipX, NtcA, and PlmA are all restricted to cyanobacteria.

Knowledge of this nitrogen regulatory interaction network of cyanobacteria, has largely benefited from the "guilty by association" principle implicit in yeast two- and three-hybrid approaches (Burillo et al., 2004; Espinosa et al., 2006; Llacer et al., 2007, 2010; Laichoubi et al., 2011, 2012; Labella et al., 2016). The same principle can be applied to proteins encoded within the same operon, particularly in the cyanobacterium Synechococcus elongatus PCC7942 (hereafter S. elongatus), where most of the mRNA transcripts identified (approximately 62\%) are monocistronic and thus the co-transcription of 2 given genes provides a very strong suggestion of functional association (Vijayan et al., 2011; Memon et al., 2013). This is the case of pipX and its downstream gene Synpcc7942_2060, called hereafter pipY in reference to its linkage to PipX in cyanobacteria.

Sequence comparisons indicate that PipY belongs to the COG0325 family, an intriguing group of highly conserved proteins that are widely distributed (Prunetti et al., 2016; and see COG0325 in the EGGNOG database ${ }^{1}$ ). The reported structure of the COG0325 yeast protein (Eswaramoorthy et al., 2003) and the structures deposited in the Protein Data Bank (PDB), without associated publication, of the products of the COG0325 genes of three additional microorganisms (Escherichia coli, Agrobacterium tumefaciens, and Bifidobacterium adolescentis; respective PDB files $1 \mathrm{~W} 8 \mathrm{G}, 3 \mathrm{R} 79$ and $3 \mathrm{CPG}$ ) indicate that these proteins are single-domain pyridoxal phosphate (PLP) binding proteins that exhibit the fold type-III of PLP-holoenzymes (Percudani and Peracchi, 2009). Clustering of COG0325 members with cell division genes in Gram-positive bacteria and mycobacteria suggested a connection with cell division in some bacterial groups (Gola et al., 2015; Prunetti et al., 2016). The genomic association of $p i p Y$ with cyanobacterial-exclusive ( $p i p X)$ and with more widely distributed bacterial genes ( $\operatorname{eep} F$ ) (Miyagishima et al., 2005), and the fact that in both cases synteny is observed in most cyanobacteria (illustrated in Figure 1), provide a strong motivation to investigate the role of COG0325 proteins in cyanobacteria in the context of paradigmatic bacterial processes such as nitrogen regulation or cell division.

Three studies addressing the characterization of $y g g S$ (encoding the E. coli COG0325 protein) null mutants revealed defects in amino/keto acid or vitamin B6 homeostasis (Ito

${ }^{1}$ http://eggnogdb.embl.de et al., 2013; Prunetti et al., 2016). The pyridoxine sensitive phenotype of $y g g S$ null mutants could be complemented by heterologous expression of the plant and human COG0325 proteins, indicating that distantly related COG0325 proteins share common functions (Ito et al., 2013). Very recently, two different reports on human epilepsy (Darin et al., 2016; Plecko et al., 2017) confirmed the involvement of PROSC (the human COG0325 protein) in vitamin B6 homeostasis, showing that loss of function mutations at PROSC are a cause of vitamin B6-dependent epilepsy.

Although our initial interest in PipY stems from its predicted involvement in the cyanobacterial nitrogen regulatory network, in the course of this study the idea that COG0325 proteins may perform the same basic functions in all types of cells gained strength. Here we show that PipY is a bona fide member of the COG0325 family performing the same basic functions previously inferred for YggS and PROSC. Genetic analyses of pipY mutants (i) confirmed phenotypes observed in E. coli yggS, such sensitivity to pyridoxine and imbalance of the amino/keto acid pools (ii) revealed new PLP-related phenotypes, including sensitivity to antibiotics targeting essential PLP-holoenzymes or synthetic lethality with cysK, and (iii) uncovered gene interactions between $p i p Y$ and pipX. We also showed that PipY overexpression increased the length of $S$. elongatus cells. Taken together, our results support a purely regulatory and universal role for COG0325 proteins, paving the way to a better understanding of these proteins and of their connections with other biological processes.

\section{MATERIALS AND METHODS}

\section{Construction of Plasmids, Cyanobacterial Strains, and Culture Conditions}

Strains and plasmids used in this work are listed in Table $\mathbf{1 .}$ Oligonucleotides used to construct plasmids and for sequencing and strain verifications are listed in Supplementary Table S1. Cloning procedures were carried out with E. coli $\mathrm{DH} 5 \alpha$, using standard techniques (Sambrook et al., 1989). All the constructs were analyzed by automated dideoxy DNA sequencing. $S$. elongatus strains were routinely grown photoautotrophically at $30^{\circ} \mathrm{C}$ while shaking under constant illumination $(40 \mu \mathrm{mol}$ photons $\mathrm{m}^{-2} \mathrm{~s}^{-1}$ ) provided by cool white fluorescent lights. Media used were blue-green algae media BG11 (BG11 $1_{0}$ plus $17.5 \mathrm{mM} \mathrm{NaNO}_{3}$ and $10 \mathrm{mM}$ HEPES/NaOH pH 7.8) and $\mathrm{BG} 11_{\mathrm{A}}$ (BG11 $1_{0}$ plus $5 \mathrm{mM} \mathrm{NH}_{4} \mathrm{Cl}$ and $5 \mathrm{mM}$ HEPES/NaOH pH 7.8). For growth on plates the media was solidified by addition of $1.5 \%(\mathrm{w} / \mathrm{v})$ agar. Plates were routinely incubated at $30^{\circ} \mathrm{C}$ under constant illumination. S. elongatus strains were transformed essentially as described by Golden and Sherman (1984).

To construct the plasmids to inactivate $p i p X$ or $p i p Y$ (Cyanobase gene ID Synpcc7942_2061 and Synpcc7942_2060, respectively), the genomic region containing the pipXY cluster was excised from pUAGC59 with $\mathrm{KpnI} / \mathrm{BamHI}$ and cloned into pGAD424, yielding plasmid pUAGC124. In parallel, a $1.4 \mathrm{~Kb}$ 
genomic region, containing the operon sequences, was amplified using primers PipXXinact-R and PipXXinact-F and cloned into pBluescriptSK+, giving plasmid pUAGC129. A 1.3 Kb fragment was excised from pUAGC129 with BamHI and cloned into pGAD424, yielding plasmid pUAGC125. The coding sequence of the cat gene was PCR-amplified from pUAGC103 with primers pairs PipX-Cm-1F/1R and 2060-Cm-1F/1R containing homologous sequences to $p i p X$ and $p i p Y$, respectively. The cat PCR products bearing either $p i p X$ or $p i p Y$ flanking sequences were used with linearized pUAGC124 (BoxI) or pUAGC125 (SacII), respectively, for recombination cloning in yeast, yielding plasmids pUAGC126 and pUAGC127.

Plasmids pUAGC126 and pUAGC127 were used, respectively, as templates for PCR amplifications with primer pairs ADCseqF/pipX-Cm-1R and 2060-Cm-1F/GBT-2R. Both PCR products together with EcoRI/KpnI restricted pGAD424 were used for recombination cloning in yeast, giving plasmid pUAGC128.

To overexpress $p i p Y$ from the IPTG-inducible Ptrc promoter, two plasmids, pUAGC290 and pUAGC294, carrying Ptrc::pipY transcriptional fusion and lacI flanked by the Neutral Site I regions of $S$. elongatus, were engineered. The pip $Y$ sequences were PCR amplified from genomic DNA using primers ORF2060-4F and 2060-3R. The amplified product was restricted with EcoRI/SalI and cloned into digested pUAGC280 and pUAGC276, yielding, respectively, plasmids pUAGC294 and pUAGC290. Plasmid pUAGC276 was obtained after amplification of DNA sequences conferring resistance to Apramycin with primers Apramicyn-1F/1R using pIJ786 as a template and the subsequent replacement of the $\mathrm{Sm}^{\mathrm{R}} / \mathrm{Sp}^{\mathrm{R}}$ cassette present in pUAGC280 by SphI/HindIII digested Apramycin cassette.

To construct a $p i p X$ overexpression plasmid $p i p X$ coding sequence was PCR amplified from genomic DNA with primers pairs PipXOV2F/PipX-3R, restricted with EcoRI/BamHI and cloned into digested pUAGC280 producing pUGAC873.

To engineer a plasmid to inactivate alr (Synpcc7942_2201), its genomic region was amplified with primers ALREcoRIF/ALRBamHI-R and the product digested with EcoRI/BamHI and cloned into restricted pGBT9 yielding plasmid pUAGC878. The coding sequence of nat gene was PCR-amplified with primers nat1ALR-F/nat1ALR-R containing homologous sequences to $a l r$. The nat PCR product was used with restricted pUAGC878 $(\mathrm{Mls})$ for recombination cloning in yeast resulting in plasmid pUAGC879.

To inactivate $p i p X$, pipY, or both simultaneously, WT S. elongatus was transformed with pUAGC126, pUGAC127 or pUAGC128, respectively. Transformants were selected with chloramphenicol $\left(2.5 \mu \mathrm{g} \mathrm{ml}^{-1}\right)$ and complete segregation was confirmed by PCR analysis with oligonucleotides pairs PipX126F/PipX5R, inter2060-1F/PipXXinact-R and PipX126F/2060Cm1F for $p i p X$, pipY and pipXY inactivations, respectively. Digestion with TasI was performed to differentiate WT ( 2 bands of $\sim 800$ and $400 \mathrm{bp}$ ) and mutant (three bands of $\sim 500,400$, and $300 \mathrm{bp}$ ) alleles. For ectopic overexpression of pipY or pipX in S. elongatus, plasmids pUAGC290 and pUAGC294 for pip $Y$ and $p U A G C 873$ for $p i p X$, were transformed and clones were selected with either apramycin $\left(10 \mu \mathrm{g} \mathrm{ml}^{-1}\right)$ or streptomycin $\left(5 \mu \mathrm{g} \mathrm{ml}^{-1}\right)$. The correct insertion into the Neutral Site was confirmed by PCR with primer pairs PTRC99Aseq-F/NSI-1F and 7942NSIA-F/NSI-1R. For constitutive increased expression of pipXY operon in S. elongatus, pUAGC410 was transformed in WT and transformants selected with kanamycin $\left(5 \mu \mathrm{g} \mathrm{ml}^{-1}\right)$.

Inactivation of cysK (gene id Synpcc7942_1466) was carried out with plasmid UGS-26-A-7 from the unigene set, an arrayed mutant library for S. elongatus (Holtman et al., 2005). Transformants were selected on kanamycin plates $(10 \mu \mathrm{g}$ $\mathrm{ml}^{-1}$ ) and complete segregation confirmed by PCR analysis using primers cysK-1F/cysK-1R. To inactivate alr (gene id Synpcc7942_2201) pUAGC879 transformants were selected on plates with nourseothricin $\left(5 \mu \mathrm{g} \mathrm{ml}^{-1}\right)$ and segregation was analyzed with primers ALREcoRI-F/ALRBamHI-R.

To construct plasmids pUAGC739 and pUAGC740 for yeast two-hybrid interaction assays, the pipY coding sequence was amplified with primers PIXX-F and PIXX-R from genomic DNA, the product restricted with EcoRI/SalI and cloned into digested pGAD424 and pBGT9. To obtain PipX and PipY derivatives carrying GAL4 (AD or BD) C-ter fusions, the $p i p X$ and $p i p Y$ coding sequences were PCR amplified from genomic DNA with primers pairs pipX-ADC-F/pipX-ADC-R and pipX-BDCF/pipX-BDC-R for $p i p X$ and pipY-ADC-F/pipY-ADC-R and pipY-BDC-F/pipY-BDC-R for pipY. PipX-ADC and PipY-ADC products were used as templates in a second round of PCR with primers ADC-F/ADC-R and similarly PipX-BDC and PipYBDC products were amplified with BDC-R/BDC-R. ADC and $\mathrm{BDC}$ amplification products were cloned, respectively, into NruI restricted pADC and pBDC yielding plasmids pUAGC745 and pUAGC746 for PipX, and pUAGC747 and pUAGC748 for PipY.

\section{PipX and PipY Immunodetection by Western Blotting in S. elongatus}

For use as immunodetection standards, pure $\mathrm{His}_{6}$-tagged S. elongatus PipX (Llacer et al., 2010) and PipY (details to be published elsewhere) were used. This last protein was the antigen utilized for preparation of the primary antibody for PipY immunodetection $\left(1.8 \mu \mathrm{g} \mathrm{ml}^{-1} \mathrm{IgG}\right.$ from anti-PipY rabbit antiserum, prepared by Genosphere Biotechnologies, Paris).

Luminescent immunodetection in western blots of homogenates of nitrate-containing liquid cultures grown to mid-exponential phase $\left(\mathrm{OD}_{750} \sim 0.5\right)$ was carried out as previously reported for PipX (Labella et al., 2016). For PipX and PipY analysis a Precellys Evolution (Bertin Technologies) glass beads homogenizer was used for cell breakage (six 25-s 6,800 rev $\mathrm{min}^{-1}$ pulses separated by $1 \mathrm{~min}$ ice-cooling intervals).

\section{RT-PCR Analysis}

RT-PCR assays were performed using $50 \mathrm{ng}$ of DNase-treated total RNA, isolated as described (Lopez-Redondo et al., 2010) from ammonium growing cells. After retrotranscription of particular mRNAs, cDNA was used as template in PCR reactions. To retrotranscribe $p i p X$, pipY and sepF, primers PipXQ82A-R, PipX-5R-X and 2059-R were used, respectively. The cDNAs were amplified with primer pairs PipXF38A-F/PipXQ82A-R (for pipX), PipX-4F/PipX-5R-X (for pipY) and 2059-F/2059-R (for sepF). $10 \mu \mathrm{L}$ of the PCR reactions were loaded in a $1.5 \%$ agarose 
TABLE 1 | Strains and plasmids.

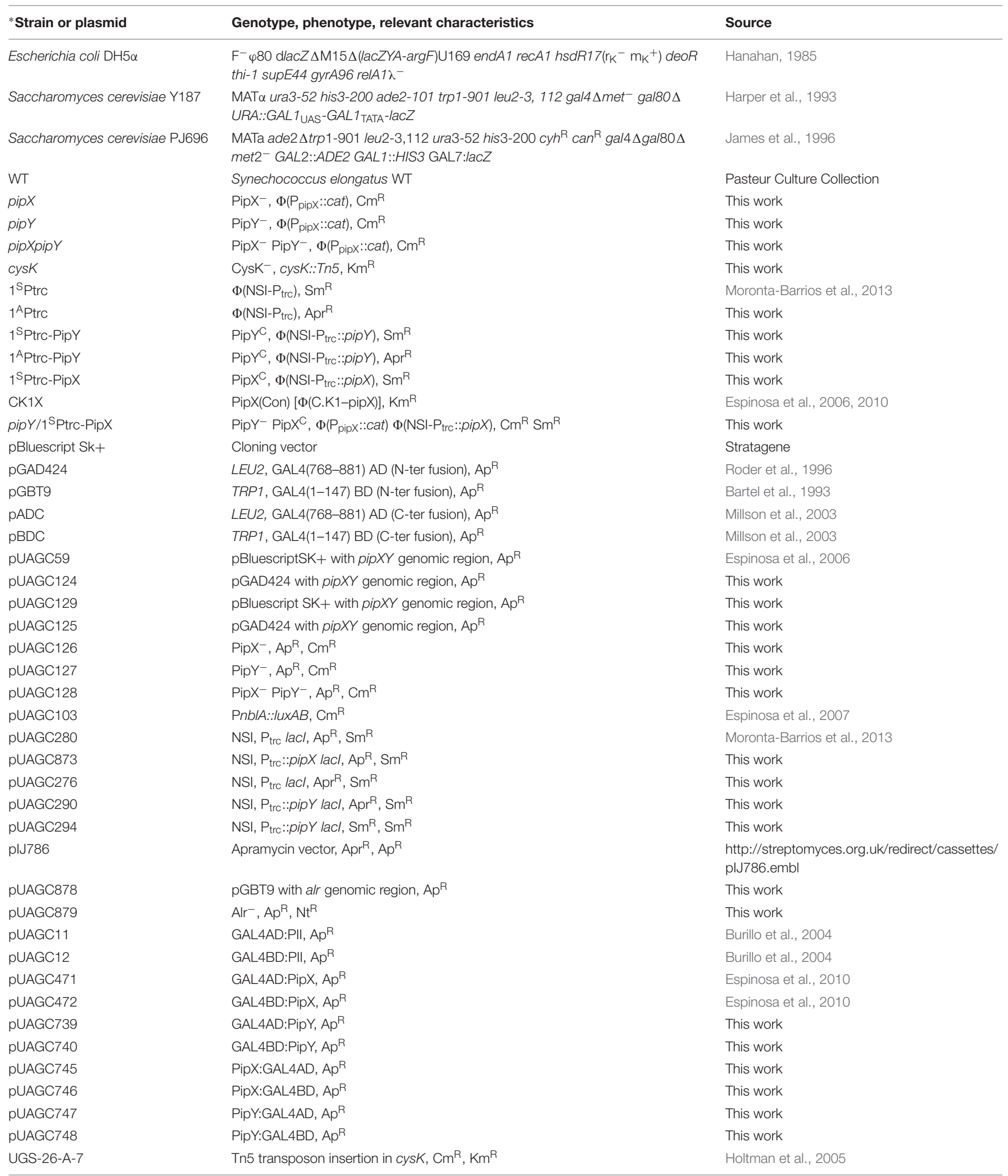

*Only fully segregated S. elongatus strains are listed. 
gel to visualize the amplified products. Housekeeping gene $r n p B$ was used as a control to verify the same input of RNA using primers rnpB-R for retrotranscription and $\mathrm{rnpB}-\mathrm{F}$ as forward primer. For co-transcription analysis of $p i p X Y$ operon, total RNA was subjected to retrotranscription using the reverse primer PipX5R-X and the cDNA used as template in a PCR reactions using the same reverse and three forward primers PipXQ34A-F, qPCR2060 and Syn2060F annealing, respectively, within the coding region of pipX, pipY and Synpcc7942_2062.

\section{Transcriptomic Analysis}

RNA was purified [phenol-chloroform extraction and TURBO DNase (Ambion) digestion] from two independent 120$\mathrm{ml}$ ammonium-containing cultures $\left(\mathrm{OD}_{750} \sim 0.7\right)$ of each S. elongatus WT and $p i p X$, $p i p Y$ or $p i p X p i p Y$ mutants. RNA was quantified spectrophotometrically. Its integrity $(\mathrm{RIN}>8)$ was proven with the Agilent RNA 6000 Nano kit. mRNA enrichment from total RNA, cDNA library construction and sequencing were carried out with an Illumina's TruSeq Stranded Total RNA with Ribo-Zero Kit and an Illumina Hiseq 2500 platform, using 100 bp paired-end sequencing reads.

Independent replicate datasets for the four S. elongatus strains, with entire genome coverage (all nucleotides covered) and 187273 average deepness, were aligned with Bowtie2 (Langmead and Salzberg, 2012) with the sequence of the S. elongatus chromosome and endogenous plasmid (Genbank entries CP000100 and CP000101, respectively), retrieving expression values for each ORF with HTseq (Anders et al., 2015), followed by differential expression analysis with R package DESeq (Anders and Huber, 2010) and hierarchical clustering and dendrogram construction with R software (R Development Core Team, 2010) and the Ape package (Paradis et al., 2004).

\section{Pyridoxine (PN) and Antibiotic Susceptibility and Protection Assays in S. elongatus}

The cells from 3-ml aliquots of the indicated $S$. elongatus strains grown to $\mathrm{OD}_{750} \sim 0.6-0.7$, suspended in $0.4 \mathrm{ml}$, were plated on solid medium supplemented when indicated with amino acids $(6.75-225 \mu \mathrm{M}$ for D-ala, $225 \mu \mathrm{M}$ for L-amino acids) or pyridoxal $(150 \mu \mathrm{M})$. A sterile disk filter was placed on the top of the plate, at the center, having been spotted with $7 \mu \mathrm{l}$ of $21.5 \mathrm{mg} \mathrm{ml}^{-1} \mathrm{PN}$ or with $4 \mu \mathrm{l}$ of one of the following antibiotics (antibiotic/concentration in $\mathrm{mg} \mathrm{ml}^{-1}$ ): apramycin/17.5, tetracycline/7, ampicillin/0.3, vancomycin/0.14, gentamycin/0.525, DCS/12.5, BCDA/12.5. After 3-days incubation at $30^{\circ} \mathrm{C}$ under constant light, the radii of the inhibition halos were measured. Plates were photographed over a white and red background, the last one to improve digital discrimination of biomass.

\section{Microscopy and Image Acquisition}

Exponential growing cells $(5 \mu \mathrm{L})$ were mounted on $1 \%$ lowmelting point agarose pads for microscopy. The samples were observed and photographed with a Leica inverted confocal microscope (running under Leica Confocal Software version
2.61, Leica Microsystems) using the HCX PL APO 63X oilimmersion objective, numerical aperture 1.4. Filter specificities for cyanobacterial auto fluorescence analysis were as follow: ex633, TD 488/543/633, em665-700. Image capture conditions were 8 bits with a $1024 \times 1024$ resolution and $5 \times$ electronic zoom.

\section{Computational Methods}

Intergenic distances between $p i p X$, $p i p Y$, sepF, and proC were calculated using their positions in the cyanobacterial genomes available on the KEGG database. A phylogenetic tree was constructed using PipX, PipY, SepF and ProC concatenated protein sequences (in the given order) with the online tool Clustal omega $^{2}$ with default parameters. When no orthologs were found in a particular genome for one or more of the four proteins, only those present were concatenated.

To measure cell length from confocal microscope images a homemade script was used. The cell measuring algorithm consisted on: (i) automatic image-blurring and thresholding parameters determination, where multiple cycles of blurring, thresholding and cell detection are computed to achieve the highest detected cells/total cells ratio; (ii) cell detection based on user input cell proportions (maximum and minimum area and length/width ratio) and shape complexity (hull area/contour area ratio) limits; (iii) determination of the rotated minimum area bounding rectangle, whose length and width are considered those of the cell.

Image J software was used to measure the radii of growth inhibition halos (ImageJ Macro is available on ${ }^{3}$ ). Color channels were separated and green and blue ones discarded. The image was thresholded with default parameters and subjected to a process of 3 cycles of dilatation, 73 cycles of erosion and 70 further cycles of dilatation to smooth the shape of the halo and to eliminate artifacts. The resultant image was adjusted to an ellipse and the diameter of the halo was calculated as the average of the major and minor axes of the ellipse minus the diameter of the disk filter.

\section{Yeast Two Hybrid Assays}

Standard yeast culture and transformation procedures were used (Ausubel et al., 1999). To determine interaction patterns among selected proteins, expression from the reporter gene lacZ in PJ696/Y187 diploids was determined in X-Gal overlay high sensitivity assays as described (Burillo et al., 2004; Labella et al., 2016).

\section{RESULTS}

\section{Genomic Context of pipY, the Cyanobacterial Gene Encoding a Member of the COG0325 Family}

To get insights into the genomic context of $p i p Y$ in cyanobacteria, we retrieved homologous sequences from all cyanobacterial

\footnotetext{
${ }^{2}$ https://www.ebi.ac.uk/Tools/msa/clustalo/

${ }^{3}$ https://dfgm.ua.es/genetica/investigacion/cyanobacterial_genetics/Resources. html
} 
genomes available in the KEGG database ${ }^{4}$. In addition to $p i p X$, two other genes, sepF and proC, were found clustering with $p i p Y$ and were also included in the in silico analyses. The genomic context of $p i p Y$ and the distances between contiguous ORFs are schematically illustrated within a phylogenetic tree constructed with the concatenated cyanobacterial sequences of these four genes (Figure 1A). Linkage between $p i p X$ and $p i p Y$ is present in most cyanobacterial genomes, with very small or non-existent distances between the two ORFs: less than $167 \mathrm{nt}$ in 71/94 cases, of which 29 show overlapping ( 1 or $4 \mathrm{nt}$ ). Thus, the genomic information indicates that co-expression and even translational coupling between $p i p X$ and $p i p Y$ may be relatively frequent amongst cyanobacteria, suggesting a strong functional connection between PipX and PipY in this phylogenetic group. In the case of S. elongatus, our results from RT-PCR (Figure 1B) and those by others (Vijayan et al., 2011) indicate that pipX and pipY form a bicistronic operon.

In $S$. elongatus and in a majority of the available cyanobacterial genomes, the genes sepF [involved in cell division and restricted to gram positive bacteria and cyanobacteria (Hamoen et al., 2006)] and proC (Pyrroline-5-carboxylate reductase, EC 1.5.1.2) (De Wergifosse et al., 1994) were found at short distances downstream of pipY. These two genes have no paralogs in S. elongatus. Although sepF and proC are monocistronic in S. elongatus (Vijayan et al., 2011; Espinosa et al., 2014) the arrangements $p i p Y$-sepF and sepF-proC were also frequently found in the available cyanobacterial genomes, suggesting that co-transcription between sepF-proC or pipY-sepF may be frequent among cyanobacteria. The clustering of these 4 genes and the occasional overlap of sepF and proC ORFs (and, to a lesser extent between $p i p Y$ and $s e p F$ ) further suggest a functional linkage between the four genes. In this context, it is worth noting that synteny of COG0325 members with both sepF and proC has been previously noted (Whitchurch et al., 1991; De Wergifosse et al., 1994). However, the precise arrangement pip Y-sepF-proC, and the linkage to the nitrogen regulatory factor $p i p X$ are cyanobacterial hallmarks.

\section{Gene Inactivation and Polar Effects within the S. elongatus pipXY Operon}

To study in vivo functions of PipY, constructs were engineered to inactivate the two genes of the pipXY operon, both individually and together. Gene inactivation was performed by allele replacement, precisely substituting the relevant coding region(s) by that of the cat gene (Supplementary Figures S1A,B). Subsequent detection by PCR and RFLP of fully segregated pipY null alleles indicated that $p i p Y$ is not essential in $S$. elongatus (Supplementary Figure S1B). Growth curves of S. elongatus strains growing with either nitrate or ammonium as nitrogen source did not show significant differences between wild type (WT) and mutant strains (Supplementary Figure S1C), indicating that lack of PipY does not affect growth in either WT or pipX backgrounds.

We wondered whether inactivation of $p i p X$ or $p i p Y$ affected transcript or protein levels from the reciprocal gene. RT-PCR

${ }^{4}$ http://www.genome.jp/kegg/
A

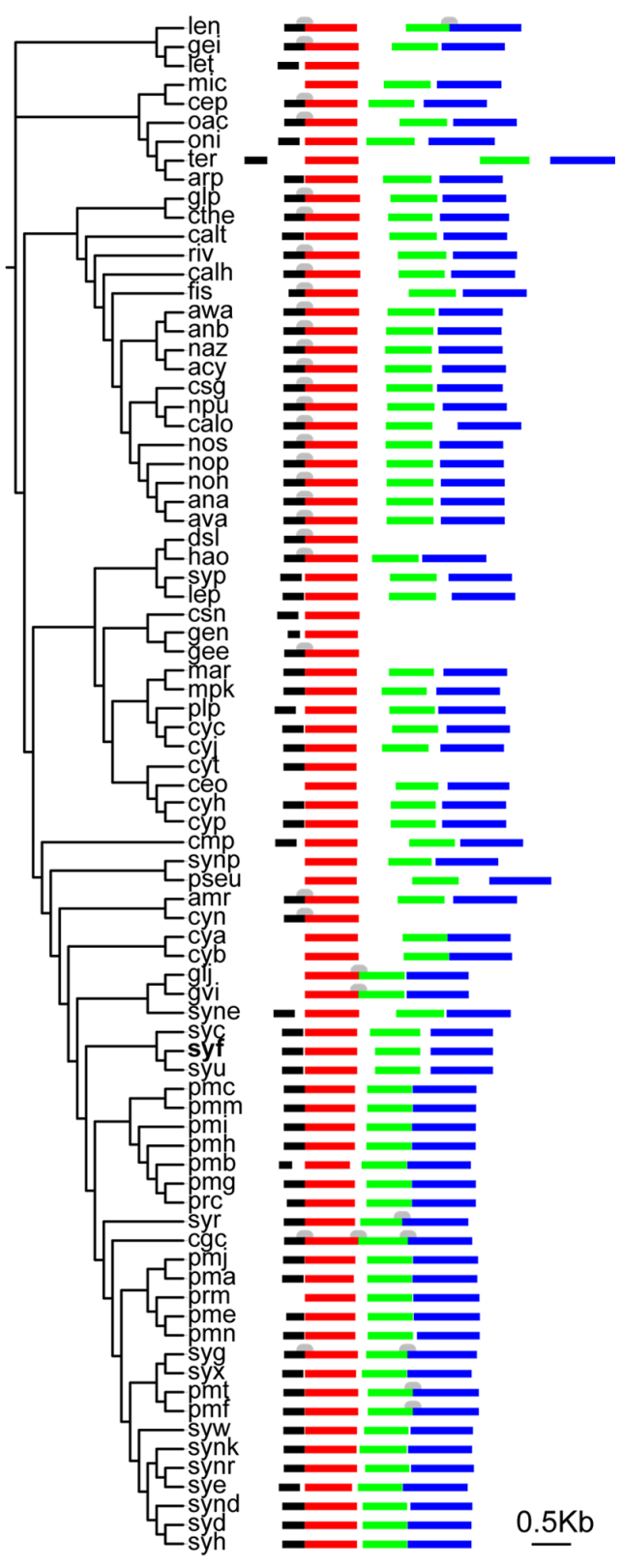

B
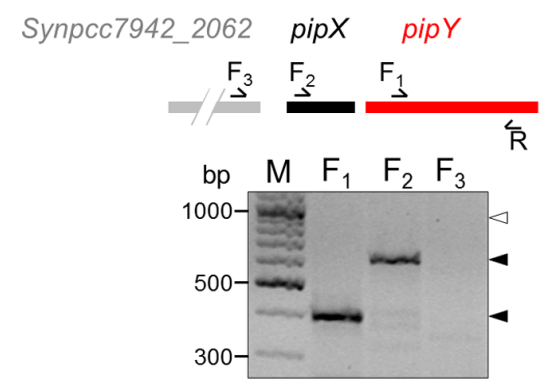

FIGURE 1 | Genomic context of the pipY gene in cyanobacteria and co-expression of pipX and pipY in S. elongatus. (A) Left, phylogenetic tree of cyanobacteria genomes available in the KEGG database. Species names

(Continued) 


\section{FIGURE 1 | (Continued)}

abbreviations are as in this database, with the one for $S$. elongatus in bold type. Right, schematic organization of the ORFs for pipX, pipY, sepF, and proC (black, red, green, and blue, respectively), with ORFs lengths and intergenic distances to scale. ORFs overlaps (1-56 bases) are indicated (gray shapes above ORFs; not to scale). In all cases genes are expressed from left to right. The thirteen species (can, cyu, mpro, scs, syj, syn, syq, sys, syt, syy syz, tel, thn) having genomes in which pipX does not cluster with pipY are omitted. (B) Co-transcription of S. elongatus pipX and pipY. The scheme on the top shows the genomic region to scale, together with locations of the oligonucleotides used in RT-PCR assays (see Materials and Methods). The photograph at the bottom shows the PCR results for each of the indicated forward primers combined with the same reverse primer $(R)$. Solid or open arrowheads indicate, respectively, the presence or absence of PCR products at the corresponding positions. M, DNA ladder, with band sizes (bp) given on the side.

showed decreased transcript levels of the non-inactivated gene of the pipXY operon, with $p i p X$ inactivation having a greater effect on $p i p Y$ transcripts than the reverse (Supplementary Figure S1D). Since the transcript levels of $s e p F$, used as an internal control, were not altered, the polar effects appear restricted to the pipXY operon. Importantly, Western blots showed that individual inactivation of $p i p X$ or $p i p Y$ decreased the levels of PipY or PipX by 15 -fold or 2-fold, respectively (Figure 2 and Table 2), suggesting that the protein levels of PipY are highly dependent on pipX gene or gene product(s). In line with this, expression from the strong CK1 promoter (strain CK1X), resulting in a significant increase in the levels of PipX (Espinosa et al., 2010), produced a 3.4fold and 4.3-fold increase in PipX and PipY, respectively (Figure 2).

\section{Phenotypic Analysis of S. elongatus pipY Mutants Reveals Universal Functions of the COG0325 Family}

Given the singular genomic context of the pipY gene, it was important to show that S. elongatus PipY is a bona fide member of the COG0325 family performing functions previously proposed or shown for other members of the family. In this context, we tried to confirm in $S$. elongatus pipY the relatively simple phenotypes reported for E. coli, as well as to explore additional phenotypic differences between mutant and WT strains of S. elongatus based in the already known structural and/or functional features of COG0325 members.

We first tested whether the pyridoxine (PN) sensitivity phenotype of the E. coli $y g g S$ null mutant and its rescue by pyridoxal (PL) or by certain L-amino acids (Prunetti et al., 2016), could also be shown in the $S$. elongatus pipY mutant by disk diffusion assays on plates. As shown in Figure 3A and Table 2, the pipY mutant was significantly more susceptible to PN toxicity than the WT strain while addition of PL decreased PN toxicity in pip $Y$ mutants and not in WT.

Next, we tried to obtain evidence of a connection between PipY and the activity of PLP-holoenzymes, since a main consequence of COG0325 deficiency appears to be the low activity of PLP-holoenzymes (Darin et al., 2016; Prunetti et al.,
2016; Plecko et al., 2017). To this end, we determined whether pipY inactivation affects the sensitivity to antibiotics targeting key PLP-holoenzymes such D-cycloserine (DCS) and $\beta$-chloro$\mathrm{D}$-alanine (BCDA), whose main target in bacteria is alanine racemase, an essential activity required for the synthesis of the cell wall (Feng and Barletta, 2003). To quantify the sensitivity of $S$. elongatus to antibiotics, we first confirmed the validity of using the square of the radius of the inhibition halo produced by different additions of antibiotic (Koch, 1999) using DCS in both WT and pipY strains. A good linear correlation between sensitivity and the amount of antibiotic added (Supplementary Figure S2) was found, thus validating the assays carried out here.

As shown in Figures 3A,B, and Table 2, the pipY mutant was more sensitive than WT to DCS and BCDA but not to control antibiotics that do not target PLP-containing proteins, including the protein synthesis inhibitors apramycin, tetracycline and gentamycin and the cell wall synthesis inhibitors ampicillin and vancomycin. Thus, the antibiotic sensitivity observed in the absence of PipY was very specific. To test if overexpression of PipY protects against DCS and/or BCDA we constructed plasmids and strains to increase PipY protein levels either constitutively or after IPTG induction (Supplementary Figure S3A). S. elongatus strain $1^{\mathrm{A}}$ Ptrc-PipY carrying Ptrc::pipY transcriptional fusion (Figure $\mathbf{3 A}$, strain abbreviated as $1 \mathrm{AY}^{\mathrm{C}}$ ) into the Neutral Site I (NSI) was tested alongside control strain $1^{A}$ Ptrc (Figure 3A, strain 1A). The 7-fold increase in PipY levels found in strain $1 \mathrm{AY}^{\mathrm{C}}$ (see Figure 2 for details) impaired growth slightly (data not shown) and resulted in rather diffuse halos, but did not increase resistance to DCS or BCDA (Figure 3A). Addition of D-Ala rescued the sensitivity of $p i p Y$ to BCDA and to a lesser extent to DCS (Figure 3C and Table 2), a result in line with the idea that BCDA is preferentially targeting alanine uptake and metabolism. Although D-Ala is known to be more effective against DCS than L-Ala in different bacteria (Wargel et al., 1970; Prosser and de Carvalho, 2013), we found that both amino acids added individually were equally effective protecting $S$. elongatus and do not show additive effects when added together (Figure 3C).

Synechococcus elongatus encodes 41 PLP-binding proteins (Percudani and Peracchi, 2009), including a putative alanine racemase, encoded by the alr gene (Synpcc7942_2201). To exclude the possibility that PipY could provide some alanine racemase activity in S. elongatus, we next tried to inactivate the alr gene in WT, pipY and $1^{\mathrm{A}}$ Ptrc-PipY strains. As expected, null alr alleles could not be completely segregated in either the WT strain or when PipY was expressed at higher levels, as in the $1^{\mathrm{A}} \mathrm{Ptrc}$ PipY strain (Supplementary Figure S3B), indicating the inability of PipY to complement Alr deficiency.

The most abundant PLP-binding proteins under standard culture conditions (Guerreiro et al., 2014) are PipY and a putative cysteine synthase (EC 2.5.1.47) encoded by cysK (Synpcc7942_1466), a non-essential gene (Rubin et al., 2015) with 3 paralogs in $S$. elongatus. Our attempts to inactivate cysK were successful in WT but not in pipY backgrounds (Figure 3D), indicating synthetic lethality between $p i p Y$ and $c y s K$, that is, both proteins contribute to essential cell functions, most likely related to the presence of the PLP cofactor. The cysK mutant was more 


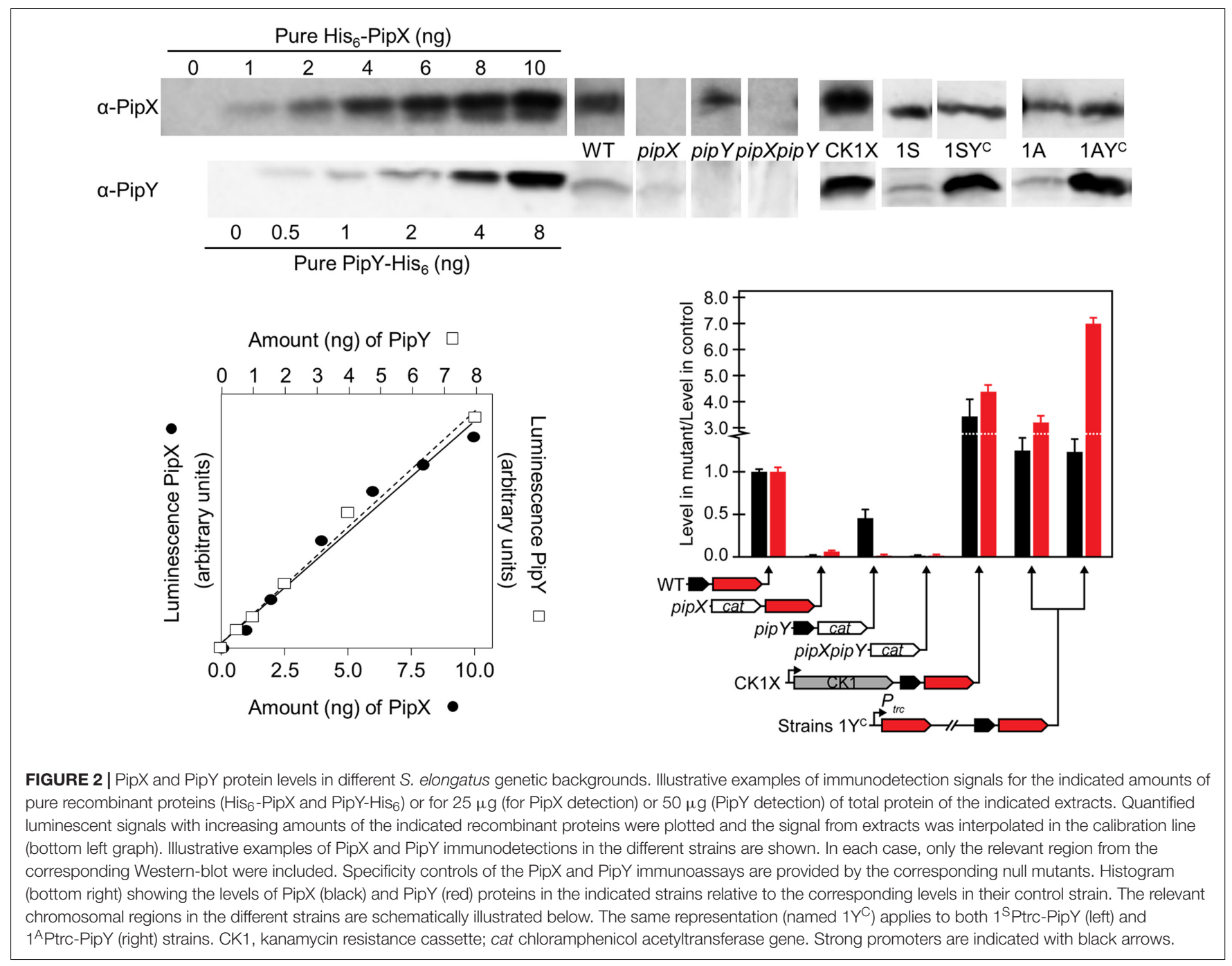

sensitive than WT but less than the pipY mutant to both DCS and BCDA (Figure 3A), indicating that PipY and to a lesser extent CysK proteins behave as non-essential targets for antibiotics DCS and BCDA in S. elongatus.

To get further insights into PipY functions in the context of amino acid-related homeostasis, the effect of addition of each of the $20 \mathrm{~L}$-amino acids on the susceptibility to DCS was determined for WT and $p i p Y$ strains. The results are summarized as a radial plot in Figure 3E. Most amino acids altered the sensitivity to DCS, indicating that they entered the cell and/or transmitted signals with metabolic consequences. The exceptions were Arg and Phe, that did not alter DCS sensitivity in any of the two strains (red and black dots coinciding with a sensitivity ratio of 1 in Figure $3 \mathrm{E}$ ), and here we cannot exclude failure of these amino acids to enter the cells in our experimental conditions. Remarkably, a previous study of amino acid uptake suggested very different permeabilities for these two amino acids in S. elongatus: while Arg uptake was negligible, Phe uptake was the highest amongst the 13 amino acids tested (Montesinos et al., 1997).
Four amino acids (Ala, Trp, Ser, and Val) exerted very large or complete protection against DCS in WT (black dots in Figure 3E) and incomplete protection in the pip $Y$ mutant (red dots in Figure 3E), indicating that a significant part of their protective effect is dependent on PipY. Nine amino acids (Gln, His, Thr, Glu, Leu, Asp, Ile, Asn, Gly) reduced but did not abolish growth inhibition by DCS to similar degrees in both strains (black and red dots near or outside the 0.5 ring but within the 1 ring). Another five amino acids (Pro, Lys, Cys, Tyr and Met) increased to different extents the sensitivity to DCS of the WT strain, while, with the exception of Met, they reduced somewhat the sensitivity of the pipY mutant, indicating that part of their toxic effect in the presence of DCS is dependent on PipY. The finding that the addition of certain amino acids produces opposite effects in WT and pipY strains of $S$. elongatus supports a complex role of PipY in amino acid homeostasis and calls attention to the diverse effects that different amino acids and metabolites can have on bacterial survival to DCS, a question of clinical relevance. 
A

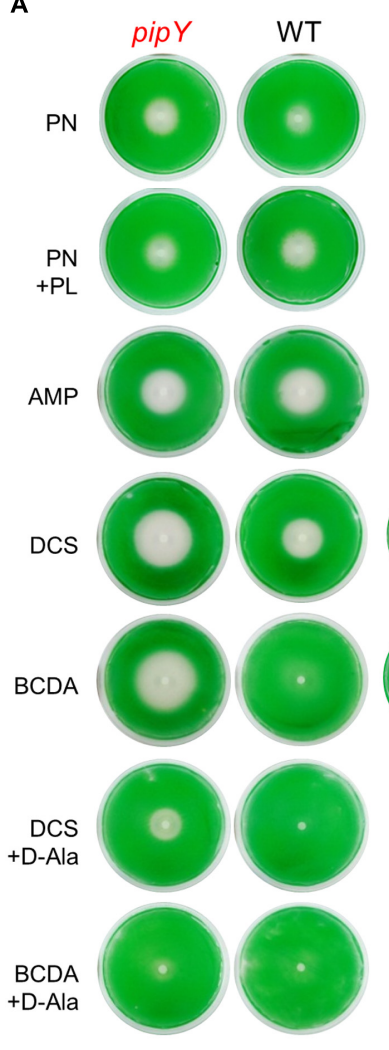

B

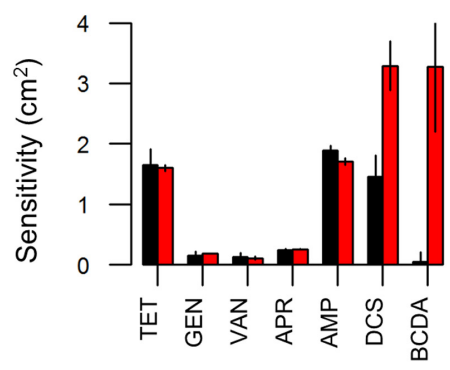

C

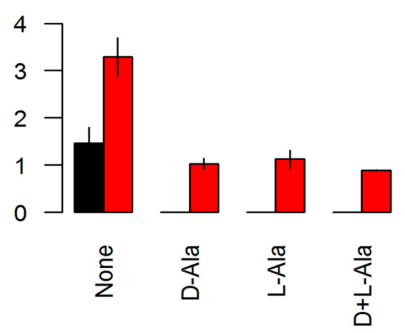

$1 \mathrm{~A} \quad 1 \mathrm{AYC} \quad$ cysK
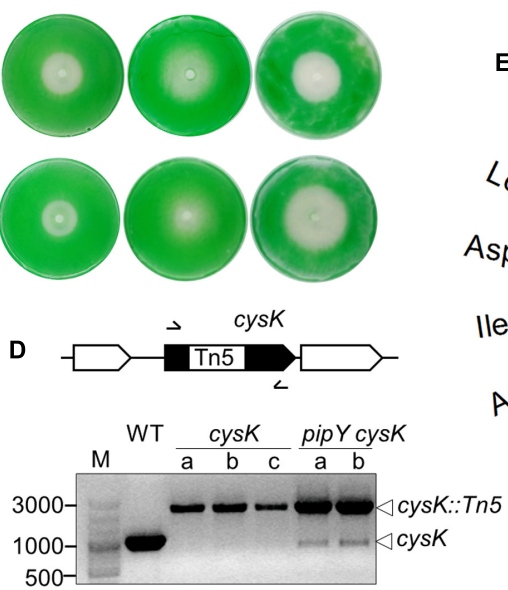

FIGURE 3 | Phenotypic features of the S. elongatus pipY mutant. (A) Representative images illustrating the sensitivity of WT (black color throughout the Figure) and pipY (red) strains to the following compounds: PN (in the absence or presence of PL), DCS or BCDA (in the absence or presence of D-Ala), and AMP. (B) Sensitivity levels for the individual antibiotics shown as bar histogram of means and standard deviations (SD) for at least two independent experiments. (C) Effect of D-Ala (0.1125 mM), L-Ala $(0.1125 \mathrm{mM})$ or both $(0.1125 \mathrm{mM}$ each) on the sensitivity of WT and pipY to DCS (Mean \pm SD, at least 2 replicates). (D) Complete and incomplete inactivation cysK in WT and pipY backgrounds, respectively. Schematic representation of the relevant $S$. elongatus region, with indication of the positions of primer pairs (arrows), and PCR analyses of up to three independently transformed clones are show. Reference size bands and amplified alleles are shown to the left and right, respectively. (E) Radial plot illustrating the influence of adding individual proteogenic L-amino acids to the culture medium on the sensitivity to DCS, expressed as the sensitivity to DCS with/without the indicated amino acid. Dotted rings indicate total protection (0), half protection (0.5), no protection (1) and twofold increase of sensitivity (2) by the corresponding amino acid. Compounds concentrations are indicated in Section Materials and Methods.

TABLE 2 | Protein levels and vitamin B6 related phenotypes of S. elongatus pipX, pipY, and pipXpipY strain.

\begin{tabular}{|c|c|c|c|c|c|c|c|c|}
\hline Strain & \multicolumn{2}{|c|}{${ }^{1}$ Protein levels } & \multicolumn{2}{|c|}{ PN sensitivity } & \multicolumn{2}{|c|}{ DCS sensitivity } & \multicolumn{2}{|c|}{ BCDA sensitivity } \\
\hline WT & $126 \pm 4$ & $70 \pm 4$ & + & + & + & - & - & - \\
\hline pipX & $0 \pm 1$ & $4 \pm 1$ & ND & ND & ++ & - & ++ & - \\
\hline pipY & $57 \pm 13$ & $0 \pm 1$ & ++ & + & +++ & + & +++ & - \\
\hline pipXpipY & $0 \pm 1$ & $0 \pm 1$ & ND & ND & ++++ & + & ++++ & - \\
\hline
\end{tabular}

${ }^{1}$ As mass $\left(\mathrm{ng} \mathrm{mg}^{-1}\right)$. Sensitivity to PN, DCS or BCDA is indicated in a qualitative scale from no (-) to high (++) sensitivity.

\section{Genetic, Not Necessarily Physical, Interactions between PipY, and PipX Revealed by Analysis of the Susceptibility of Mutants to DCS and BCDA}

Once we obtained in vivo evidence indicating that PipY is a typical COG0325 protein, functioning in amino/keto acid and vitamin B6 homeostasis, we explored the PipY connection with the nitrogen regulator PipX in the context of the susceptibility to PLP-targeting antibiotics. To this end, we determined the susceptibility of pipX and pipXpipY mutants to DCS, BCDA and other antibiotics used as controls. As shown in Figure 4A, inactivation of pipX increased sensitivity to at least BCDA in WT (compare WT and $p i p X$ ) and to at least DCS in pip $Y$ backgrounds (compare $p i p Y$ and $p i p X p i p Y$ ) without affecting sensitivity to 
A
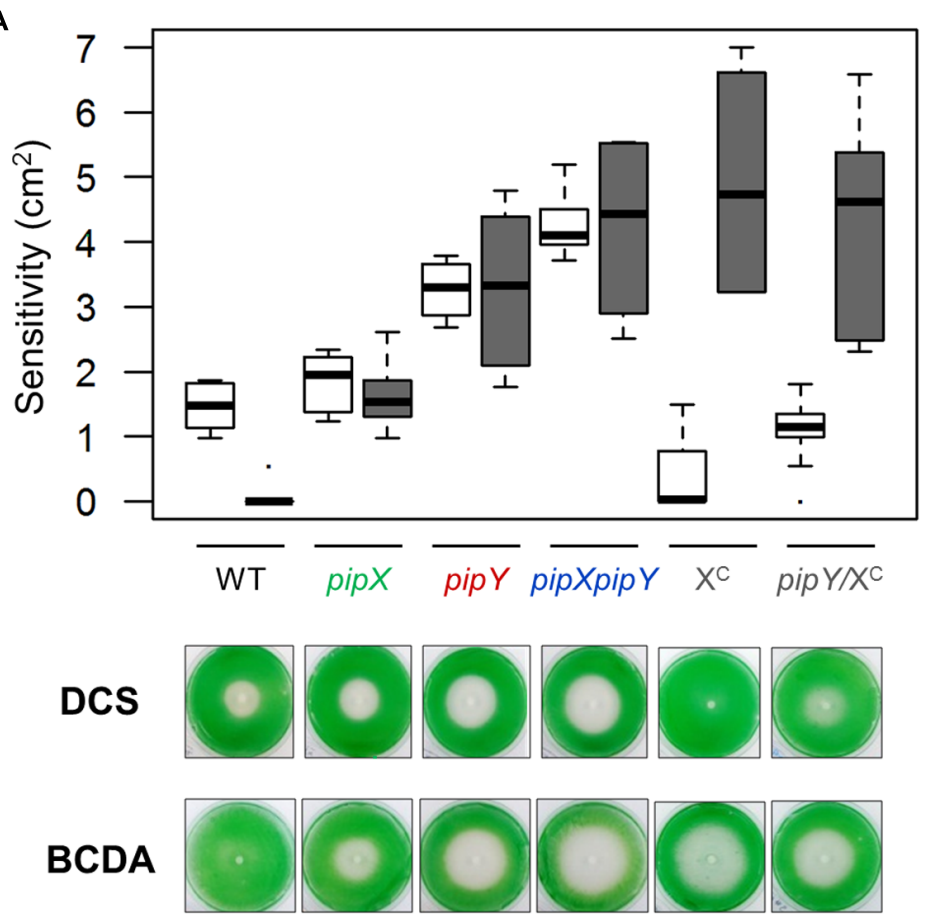

B
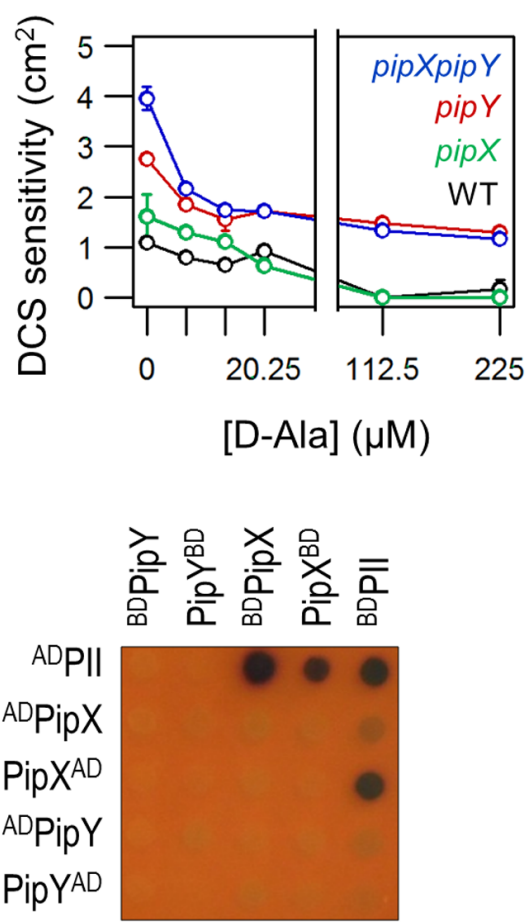

FIGURE 4 | Interactions between PipY and PipX (A) Top, boxplot (median, box from first to third quartile, Tukey whiskers and outliers represented by dots) of DCS (white) and BCDA (gray) sensitivities for the indicated strains. In each case, a minimum of 3 independent experiments with 2 or more replicates were performed. Bottom, representative photographs illustrating the sensitivity of the indicated strains to DCS and BCDA. (B) Effects of different concentrations of D-Ala on the sensitivity to DCS (Mean \pm SD, 2 replicates). (C) Representative example of yeast 2-hybrid interaction assays between the indicated proteins (see text for details). Yeast diploids were supplemented with X-gal to inform on lacZ reporter after 4 days of incubation on replete medium.

the control antibiotics (Supplementary Figure S4), thus revealing gene interactions between $p i p X$ and $p i p Y$.

The greater DCS sensitivity of pipXpipY versus $p i p Y$ suggested that PipX can protect against DCS in a PipY independent manner. To obtain additional evidence for this, we determined the effect of increasing PipX levels in the absence of PipY. To this end, we previously constructed $1^{\mathrm{S}}$ Ptrc-PipX (abbreviated as $\mathrm{X}^{\mathrm{C}}$ ) and $p i p Y / 1^{\mathrm{S}}$ Ptrc-PipX (abbreviated as pip $Y / \mathrm{X}^{\mathrm{C}}$ ) strains (Supplementary Figure S3C). Suppression of the DCS hypersensitivity by overexpressing PipX and no significant effect on the BCDA phenotype (Figure 4A) indicated that an excess of PipX compensates for the lack of PipY in the presence of DCS, but not in the presence of BCDA. Moreover, addition of D-Ala allowed complete protection against DCS in WT and pipX strains and partial protection in $p i p Y$ or pipXpipY strains (Figure 4B). Where complete or partial protection by D-Ala was obtained, protection was already significant at concentrations of D-Ala as low as $6.75 \mu \mathrm{M}$, and maximal at concentrations of 112-225 $\mu \mathrm{M}$. Therefore, some PipY protein appears to be required for the protective effect of D-Ala (Figure 4) or L-Ala (Figure 3) in the presence of DCS, a result emphasizing the metabolic imbalance of the pipY mutant.

To investigate whether PipX and PipY may interact physically with each other, we used the yeast 2-hybrid system. Because artifacts such as occlusion of interaction surfaces by GAL domains occasionally result in false negatives, we tested up to four different pairwise combinations of PipX and PipY fusion proteins, in order to maximize the possibilities of detecting interaction signals. We constructed PipY fusions to N-terminally located GAL4AD ( ${ }^{\mathrm{AD}}$ PipY) or GAL4BD ( $\left.{ }^{\mathrm{BD}} \mathrm{PipY}\right)$ domains as well as PipY (PipY ${ }^{A D}$ and $\left.\operatorname{PipY}^{\mathrm{BD}}\right)$ and PipX $\left(\mathrm{PipX}^{\mathrm{BD}}\right)$ fusions to C-terminally located domains of GAL4 and performed assays with additional PipX ( ${ }^{\mathrm{AD}} \mathrm{PipX}$ and $\left.{ }^{\mathrm{BD}} \mathrm{PipX}\right)$ and PII ( ${ }^{\mathrm{AD}} \mathrm{PII}$ and $\left.{ }^{\mathrm{BD}} \mathrm{PII}\right)$ fusions, the later ones to provide positive controls. Interaction signals were detected by the control pairs PII-PII and PII-PipX, but not for PipX-PipX, PipY-PipY or PipX-PipY (Figure 4C). While the lack of self-interaction signals agrees with the monomeric nature of both PipX and PipY, the lack of interactions signals from the four PipX-PipY combinations tested indicate that these proteins do not interact in the yeast system.

\section{Transcript Analysis Reveals Functional Connections between PipY and the Co-regulator PipX}

Next, we explored interactions between PipY and PipX in the context of transcriptional regulation. PipX, known as the co-activator of NtcA, is involved in a wider regulatory network, affecting multiple functions in S. elongatus (Espinosa et al., 2014) and we wondered whether PipY may play a role in connection 
with the transcriptional functions of PipX as a global regulator of gene expression. To explore this idea, we compared transcript profiles of WT, $p i p X$, pipY and pipXpipY strains. Because of the polar effects observed at the pipXY operon (Figure 2 and Supplementary Figure S1D), interpretation of orthodox mutant/WT comparisons appears to be straightforward for the double pipXpipY mutant but not for the single ones, since the $p i p X$ null strain accumulates very little PipY protein. However, by performing $p i p Y / p i p X p i p Y$ or $p i p X / p i p X p i p Y$ comparisons we can focus on the effects provided by the presence of PipX or PipY proteins, even if at a low level, in the absence of the other.

Differentially expressed genes scoring above the cutoff for a $\log 2$ fold change in any of the pipY/pipXpipY, pipX/pipXpipY or WT/pipXpipY comparisons were selected. This set of 78 genes was hierarchically clustered according to their expression patterns (Figure 5A and Supplementary Table S2). Transcripts up-regulated in any of the three comparisons, for which positive regulation (by PipX and/or PipY) could be inferred, doubled in number to those downregulated. One third (17 out of 51) of the transcripts activated by PipX and/or PipY were NtcA targets, of which 15 clustered together in a group defined by upregulation in the WT/pipXpipY comparison [Cluster I, containing only canonical NtcA activated genes (Espinosa et al., 2014)]. Cluster I contains two sub-clusters with expression patterns consistent with PipX and PipY having independent (IA) or additive (IB) effects, respectively. In contrast, Cluster II contained only two NtcA targets, one of which was gifA, a gene repressed by $\mathrm{NtcA}$ and subjected to complex regulation (Garcia-Dominguez et al., 2000; Galmozzi et al., 2007; Espinosa et al., 2014; Klahn et al., 2015). Cluster II expression patterns are characterized by upregulation in the $p i p X / p i p X p i p Y$ comparison (Cluster IIA) or in both $p i p X / p i p X p i p Y$ and $p i p Y / p i p X p i p Y$ comparisons (Group IIB). While the larger Cluster IIA is relatively heterogeneous, Cluster IIB shows a rather uniform pattern consistent with independent activation by PipX and PipY and no activation when both proteins are co-expressed, suggesting that PipX and PipY can interfere with each other functions. Interestingly, the expression patterns of Clusters II and III (were no NtcA targets were found) appear inverted, in particular their more homogeneous subgroups B, with Cluster IIIB characterized by down regulation in both $p i p X / p i p X p i p Y$ and $p i p Y / p i p X p i p Y$ comparisons.

To further investigate the internal coherence of the groups of genes obtained by hierarchical clustering we followed the COG (cluster of orthologous genes) classification system to assign functions and compare the distribution of COG categories and of genes of unknown function amongst the 3 clusters (Figure 5B). Genes of unknown function made similar contributions to the complete S. elongatus genome (ca. 37\%) and to the 78 genes included in the 3 clusters analyzed here (ca. 36\%), but were slightly overrepresented in Cluster II (12 out of 28; ca $43 \%)$ and underrepresented in Clusters I (30\%) and III (33\%). Inorganic ion transport and metabolism genes were overrepresented ( $16 \%$ versus $4 \%$ in the complete S. elongatus genome), being most abundant in Cluster I (14 out of 23; $39 \%)$. The category post-translational modification, protein turnover, chaperones was found exclusively in Cluster III (7 out of $27 ; 25 \%$ ), while coenzyme transport and metabolism genes were more represented in Cluster II (5 out of $28 ; 17 \%$ ). Amino acid transport and metabolism was the only category appearing in all three clusters with a similar abundance (2 out of 23,3 out of 28 and 2 out of 27 for Clusters I, II and III, respectively). The remaining categories were represented

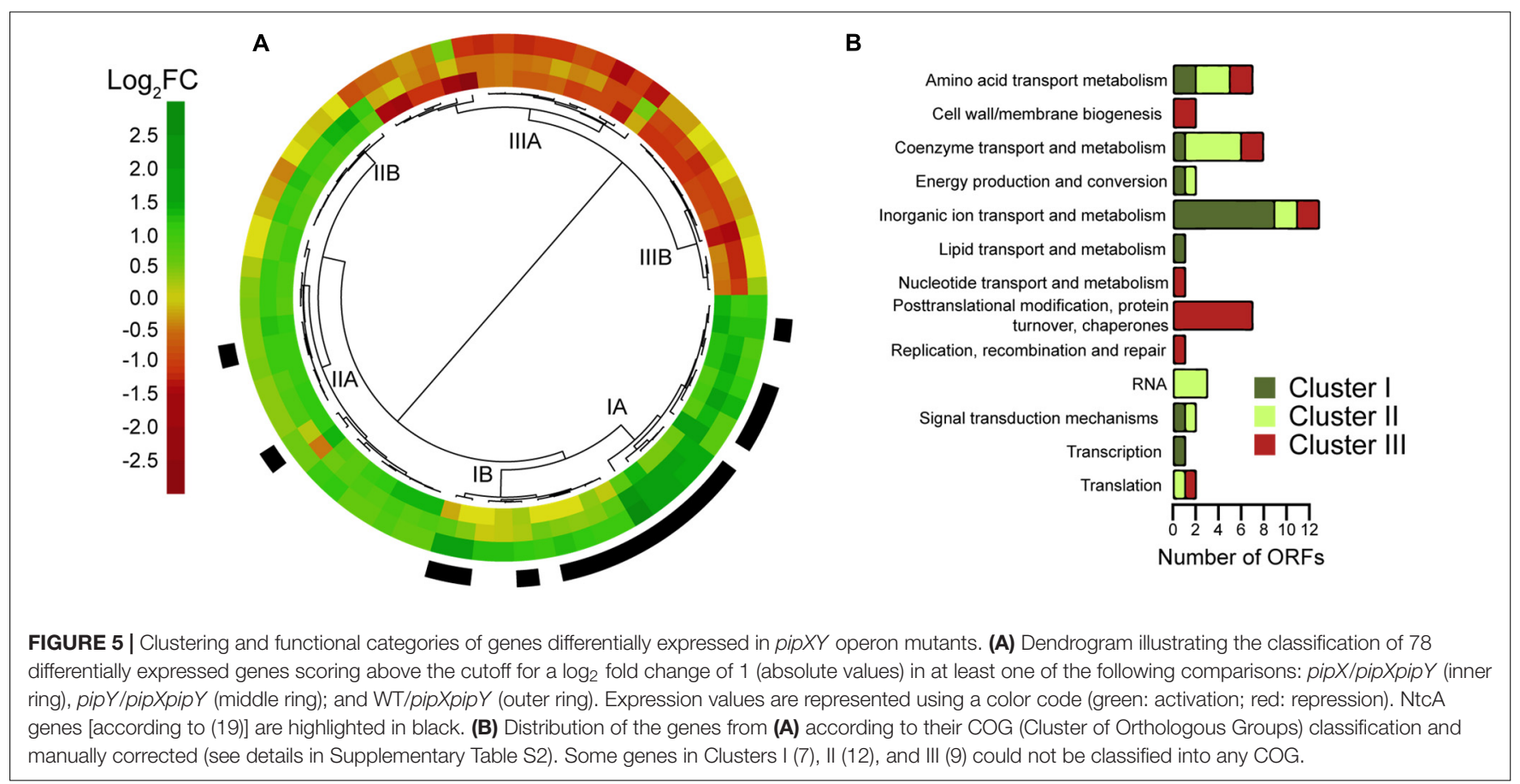


in just one of the clusters and contained a maximum of 2 genes.

\section{PipY Overexpression Increases S. elongatus Cell Length}

The strong pipY-sepF synteny in cyanobacteria made us wonder whether S. elongatus PipY has a role on the regulation of cell division or cell size. Since $p i p Y$ cells did not show the filamentous phenotypes obtained by depletion of proteins with structural roles in cell division (Figure 6), we investigated whether higher levels of PipY would result in a cell division related phenotype. To this end, we used S. elongatus strains (Supplementary Figure S3A) expressing pipY from a Ptrc promoter in an ectopic location (NSI). This allowed us to increase the levels of PipY either constitutively or to much higher levels after IPTG induction. Strains $1^{\mathrm{S}}$ Ptrc-PipY and $1^{\mathrm{A}}$ Ptrc-PipY (in reference to selection markers $\mathrm{Sm}^{\mathrm{R}}$ or $\mathrm{Apr}^{\mathrm{R}}$, respectively) were tested alongside control strains $1^{\mathrm{S}} \mathrm{Ptrc}$ and $1^{\mathrm{A}} \mathrm{Ptrc}$. As shown in Figure 2, strains $1^{\mathrm{S}} \mathrm{Ptrc}$ PipY and $1^{A}$ Ptrc-PipY allowed moderate increases of PipY levels ( $\sim 4$-fold and $\sim 7$-fold over the WT levels, respectively) in the absence of IPTG.

Cells from exponentially growing cultures were observed by Laser Scanner Confocal Microscopy and their length measured. As shown in Figure 6, elimination of PipY or a $\sim 4$-fold increase over the WT levels did not alter cell dimensions in a significant way, while larger increases in the PipY levels correlated with larger cells. A $\sim 7$-fold increase in PipY resulted in a $14.4 \%$ of increase in the average cell length of S. elongatus. Further overexpression in the presence of IPTG resulted in a $28 \%$ increase in the average cell length.

\section{DISCUSSION}

Our genetic analyses in $S$. elongatus show that $p i p Y$ is a typical member of the COG0325 family of PLP-binding proteins recruited to the cyanobacterial nitrogen regulatory network. Phenotypic analyses with the pipY mutant demonstrated the involvement of PipY in amino/keto acid and PLP homeostasis. In addition, work with $p i p X$ and $p i p Y$ mutant and derivative strains revealed gene interactions in the contexts of survival to PLP targeting antibiotics and of transcriptional regulation, placing PipY in the same genetic pathway as PipX, a protein interacting with 2-OG sensors in cyanobacteria.

Several lines of evidence support the universality of COG0325 functions, suggesting a regulatory rather than an enzymatic function for COG0325 proteins. The pyridoxine toxicity phenotype reported for E. coli yggS (Prunetti et al., 2016) could be rescued by heterologous expression of PROSC (Darin et al., 2016) and here we show that the S. elongatus pipY mutant is also hypersensitive to pyridoxine. Importantly, despite the fact that the Val excretion phenotype reported for the E. coli MG1655 yggS mutant (Ito et al., 2013) was not confirmed for E. coli BW25133 yggS (Prunetti et al., 2016) it could nevertheless be rescued by expressing the plant or human (named PROSC) COG0325 proteins (Ito et al., 2013). Thus, distantly related COG0325 proteins can contribute to metabolic homeostasis in different

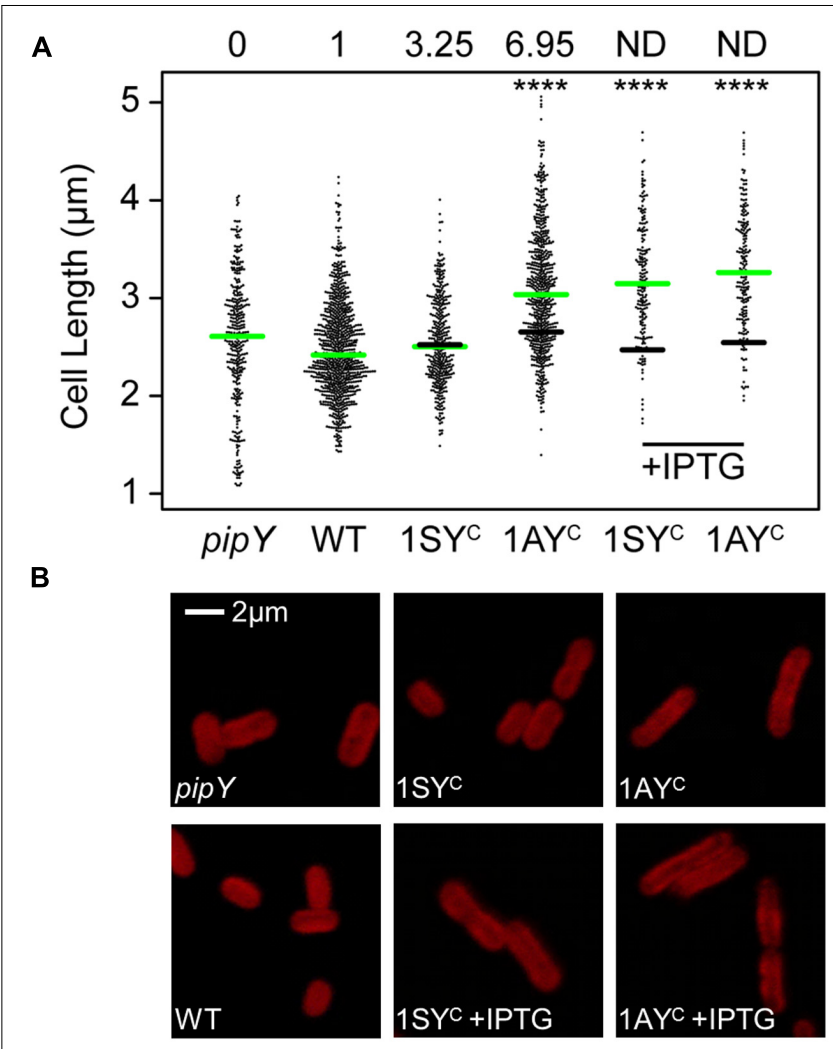

FIGURE 6 | Effects of PipY protein levels on S. elongatus cell length. (A) Scatterplot of cell lengths for the indicated strains. The median for each strain is indicated with a green line. For $1 \mathrm{AY}^{\mathrm{C}}$ and $1 S Y^{\mathrm{C}}$ strains $\left(1^{\mathrm{A}} \mathrm{Ptrc}-\mathrm{PipY}\right.$ and $1^{\mathrm{S}} \mathrm{Ptrc}-\mathrm{PipY}$, respectively) the black line indicates the median of their respective control strain ( $1^{A} \mathrm{P}$ trc and $1^{\mathrm{S}} \mathrm{Ptrc}$, respectively). The minimum number of cells measured was $n=186$. Wilcoxon rank sum test analysis of cell length data produced $p$-values $<0.0001(* * * *)$. PipY protein levels relative to that in their control are indicated for each strain on the top.

(B) Representative cell micrographs from strains in (A). Pictures were taken after $24 \mathrm{~h}$ of incubation. Where indicated, IPTG was used at $1 \mathrm{mM}$, final concentration.

cell types, rescuing species (or even strain) -specific defects. It is worth noting that the lack of in vitro evidence of amino acid racemase, decarboxylase, deaminase or transaminase activities from the E. coli, B. subtilis or human proteins (Ito et al., 2013), and the lack of viability of alr mutants in S. elongatus, even in the presence of increased levels of PipY, argue against these proteins having enzymatic functions.

The high reactivity of PLP implies that cells must have mechanisms to keep the intracellular level of PLP low while supplying enough PLP for the newly synthesized apoB6 enzymes to become active. Amongst these mechanisms are the effective feedback inhibition by PLP and its tight binding to the producing enzymes PNP oxidase, PL kinase and PLP synthase [(Ghatge et al., 2012) and references within] and the ability of PLP-dependent enzymes to trigger product release from the PLP synthase (Moccand et al., 2011). To cope with the PLP derived toxicity and optimize the delivery of the cofactors to PLP-apoenzymes, some PLP-binding 
proteins would have evolved to act as PLP reservoirs and PLP delivering modules for the essential apo-enzymes. It is worth noting that, in contrast to the situation in type III enzymes, the PLP cofactor of COG0325 proteins is solvent exposed [discussed in Ito et al. (2013)], and thus appears to be appropriately placed for the proposed roles on PLP homeostasis.

The synthetic lethality observed here between S. elongatus $p i p Y$ and $c y s K$ has a precedent in the conditional synthetic lethality reported for $E$. coli $y g g S$ and $g l y A$ (Nichols et al., 2011; Prunetti et al., 2016). The inferred functional redundancy between COG0325 proteins and two different PLP-holoenzymes strongly suggests that CysK and GlyA also contribute to PLP homeostasis in S. elongatus and E. coli, respectively. Functional redundancy amongst PLP-holoenzymes appears to occur even in organisms with relatively small genomes, as $S$. elongatus, where out of the 41 PLP-binding protein sequences found (Percudani and Peracchi, 2009), 11 corresponded to non-essential and 6 to beneficial genes (their inactivation slows growth of cultures) under standard photoautotrophic conditions (Guerreiro et al., 2014; Rubin et al., 2015). Functional redundancy between the regulatory COG0325 proteins and PLP-holoenzymes, revealed by synthetic lethality in two distantly related bacteria, suggest that at least some of the multiple PLP-containing proteins expressed in leaving cells participate in PLP homeostasis. It also explains that, despite the universality of the PLP-derived challenges in all types of cells, COG0325 proteins are neither essential (in the so far characterized systems), nor ubiquitous (Prunetti et al., 2016).

The main mechanism of resistance to DCS in bacteria is overexpression of the essential protein alanine racemase (Caceres et al., 1997). In contrast, PipY overexpression did not increase resistance to DCS or BCDA in $S$. elongatus. This finding, and the implication of PipX in survival to DCS indicates a very different basis for the protective role of PipY against DCS, supporting the idea that PipY protects the essential and high affinity targets against DCS indirectly, by affecting the activity of PLP holo-enzymes. In this context, there are precedents for regulatory genes involved in basic metabolic processes whose inactivation alters bacterial susceptibility to antibiotics (Martinez and Rojo, 2011). In S. elongatus PipX and/or PipY would contribute to increasing the intracellular levels of DCS antagonists, that is, alanine or related amino acids. It is worth noting that inactivation of L-alanine dehydrogenase is also a mechanism for DCS resistance (Desjardins et al., 2016) and that nitrogen specific responses have been implicated in the control of alanine metabolism (Lahmi et al., 2006).

Given the regulatory complexity involving PLP-holoenzymes and central metabolism, it now seems naive to expect that COG0325 deficiency caused accumulation or depletion of the same metabolite(s) in different experimental conditions or cell types. However, the prediction is that COG0325 defective mutants would be impaired in the regulation of biological pathways or processes responsive to amino/keto acids. One of such processes is the nitrogen regulatory network of cyanobacteria, which is integrating and transmitting signals of the 2-OG levels, the main indicator of the nitrogen/carbon balance in these organisms.

Two lines of results place PipX and PipY within the same genetic pathway. In particular, both proteins make contributions to resistance to PLP-targeting antibiotics DCS or BCDA and to expression of a common set of transcripts. The observed genetic interactions between PipY and PipX are best explained by PipY affecting the levels of the amino/keto acids relevant in those biological pathways and, although physical interactions between PipX and PipY proteins in S. elongatus can not be ruled out at this stage, exhaustive yeast two-hybrid interaction assays did not support it.

The similar effects of PipX and PipY on NtcA targets (Cluster I in Figure 5) suggest that PipY may have a positive effect on the intracellular levels of 2-OG under the experimental conditions used here. The subdivision of Cluster I transcripts into two subclusters with expression patterns suggestive of PipX and PipY having independent (IA) or additive (IB) effects, respectively, can be rationalized on the bases of the affinity of NtcA for

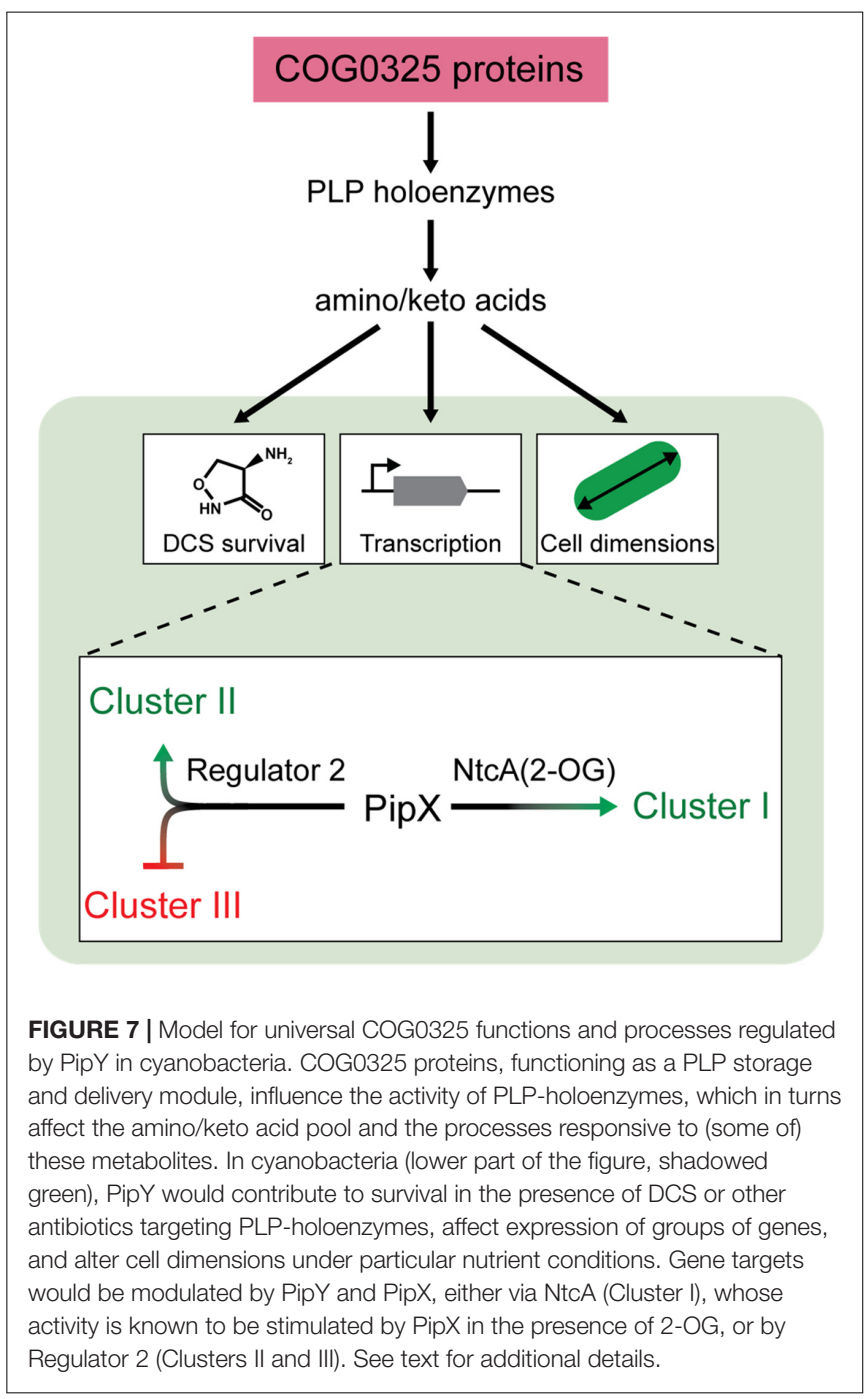


its different binding sites (Espinosa et al., 2007; Forcada-Nadal et al., 2014). NtcA targets from Cluster IB would provide weaker NtcA binding and thus greater dependency on both PipX and PipY. The transcriptome-based genetic analysis performed here supports previous work indicating that PipX modulates a large set of genes that also include NtcA-independent target genes (Espinosa et al., 2014; Labella et al., 2016) and further suggest that at least two types of transcriptional complexes are co-regulated by PipY and PipX (Figure 7). One would correspond to the already characterized NtcA(2-OG)-PipX complexes and the other one, defined by Clusters II and III, would require the implication of a second transcriptional regulator exerting opposite effects in Clusters II and III ("Regulator 2" in Figure 7). PlmA, the only other transcriptional regulator known to interact with PipX (Labella et al., 2016), is so far the best candidate for Regulator 2. It follows that the expression of Clusters II and III could also be affected by 2 -OG or related metabolite(s) whose levels would be influenced by PipY.

Synteny between $y \operatorname{lmE}$, the gram-positive ortholog of pipY, and cell division genes or cell division clusters has been the subject of discussions (Gola et al., 2015; Prunetti et al., 2016), but there are only two reports assigning cell size or cell wall related phenotypes to $y l m E$ null or overexpressing strains. Streptococcus pneumoniae ylmE cells were slightly larger (Fadda et al., 2003), while a B. subtilis strain overexpressing YlmE was blocked in biofilm formation (Kolodkin-Gal et al., 2010). In addition, no phenotype, that is, normal cell dimensions under standard culture conditions have been reported for E. coli yggS (Prunetti et al., 2016) and found here for S. elongatus pipY. However, genetic alterations causing more drastic alterations of amino/keto acid homeostasis, such as presumably double mutations ( $y g g S$ glyA) in E. coli (Prunetti et al., 2016) and overexpression of PipY increased cell length up to $23 \%$ or $28 \%$, respectively. These findings support the notion that perturbations of the amino/keto acid pool may result in the accumulation of metabolic signals controlling processes related to cell wall metabolism or cell size. In this context, the best characterized example of a nutrientdependent pathway coordinating cell division and cell size with growth rate (using UDP-glucose as a molecular proxy) is that of the Gram-positive model organism B. subtilis (Chien et al., 2012). In the case of $S$. elongatus, a link between particular nutrient conditions (combined phosphorus limitation and abundancy of reduced nitrogen) and morphological flexibility has been established (Goclaw-Binder et al., 2012), raising questions on whether this phenomenon relays on amino/keto acids signals.

\section{REFERENCES}

Anders, S., and Huber, W. (2010). Differential expression analysis for sequence count data. Genome Biol. 11:R106. doi: 10.1186/gb-2010-11-10-r106

Anders, S., Pyl, P. T., and Huber, W. (2015). HTSeq-a Python framework to work with high-throughput sequencing data. Bioinformatics 31, 166-169. doi: 10.1093/bioinformatics/btu638

Ausubel, F. M., Brent, R., Kingston, R. E., Moore, D. D., and Seidman, J. G. (eds). (1999). Short Protocols in Molecular Biology. New York, NY: John Wiley \& Sons, Inc.
In summary, the results presented here provide important insights into the COG0325 family of proteins and the universality of their functions, clearly interconnected with the activity of PLP-holoenzymes. Work with PipY, the cyanobacterial member of this widespread family, confirms the conserved roles in vitamin B6 and amino/keto acid homeostasis and extends our functional understanding of COG0325 proteins. We also provide genetic evidence for the recruitment of PipY into the 2-OGdependent nitrogen interaction network. This work emphasizes the regulatory importance of COG0325 proteins in central metabolism and suggest their implication in the metabolicdependent coordination of cell size.

\section{AUTHOR CONTRIBUTIONS}

Designed research: AC, JL, RC, JE, and VR. Performed research: JL, RC, JE, VR, and AF-N. Analyzed data: AC, JL, RC, JE, AF-N, and VR. Wrote the paper: AC. Contributed new reagents or analytic tools: JL.

\section{FUNDING}

This work was supported by grants from the Valencian (Gerónimo Forteza contract FPA/2015/052 to JL; PrometeoII/2014/029 to VR) and Spanish Governments (BFU2012-33364 and BFU2015-66360-P to AC; BFU2014-58229P to VR).

\section{ACKNOWLEDGMENTS}

We thank C.V. Racovac, A. Llop, K. Laichoubi, MA. Castells and JL. Llacer, for technical and experimental support, S.S. Golden for gift of plasmid UGS-26-A-7, R. Percudani/A. Peracchi for introducing S. elongatus into the B6 database (http://bioinformatics.unipr.it/B6db), F. Rodriguez-Mateos and R. Dixon for constructive insights and discussions.

\section{SUPPLEMENTARY MATERIAL}

The Supplementary Material for this article can be found online at: http://journal.frontiersin.org/article/10.3389/fmicb. 2017.01244/full\#supplementary-material

Bartel, P., Chien, C. T., Sternglanz, R., and Fields, S. (1993). Elimination of false positives that arise in using the two-hybrid system. Biotechniques 14, 920-924.

Burillo, S., Luque, I., Fuentes, I., and Contreras, A. (2004). Interactions between the nitrogen signal transduction protein PII and N-acetyl glutamate kinase in organisms that perform oxygenic photosynthesis. J. Bacteriol. 186, 3346-3354. doi: 10.1128/JB.186.11.3346-3354.2004

Caceres, N. E., Harris, N. B., Wellehan, J. F., Feng, Z., Kapur, V., and Barletta, R. G. (1997). Overexpression of the D-alanine racemase gene confers resistance to D-cycloserine in Mycobacterium smegmatis. J. Bacteriol. 179, 5046-5055. doi: $10.1128 /$ jb.179.16.5046-5055.1997 
Chien, A. C., Hill, N. S., and Levin, P. A. (2012). Cell size control in bacteria. Curr. Biol. 22, R340-R349. doi: 10.1016/j.cub.2012.02.032

Darin, N., Reid, E., Prunetti, L., Samuelsson, L., Husain, R. A., Wilson, M., et al. (2016). Mutations in PROSC disrupt cellular pyridoxal phosphate homeostasis and cause vitamin-B6-dependent epilepsy. Am. J. Hum. Genet. 99, 1325-1337. doi: 10.1016/j.ajhg.2016.10.011

Desjardins, C. A., Cohen, K. A., Munsamy, V., Abeel, T., Maharaj, K., Walker, B. J., et al. (2016). Genomic and functional analyses of Mycobacterium tuberculosis strains implicate ald in D-cycloserine resistance. Nat. Genet. 48, 544-551. doi: 10.1038/ng.3548

De Wergifosse, P., Jacques, B., Jonniaux, J. L., Purnelle, B., Skala, J., and Goffeau, A. (1994). The sequence of a $22.4 \mathrm{~kb}$ DNA fragment from the left arm of yeast chromosome II reveals homologues to bacterial proline synthetase and murine alpha-adaptin, as well as a new permease and a DNA-binding protein. Yeast 10, 1489-1496. doi: 10.1002/yea.320101113

Espinosa, J., Castells, M. A., Laichoubi, K. B., Forchhammer, K., and Contreras, A. (2010). Effects of spontaneous mutations in PipX functions and regulatory complexes on the cyanobacterium Synechococcus elongatus strain PCC 7942. Microbiology 156, 1517-1526. doi: 10.1099/mic.0.037309-0

Espinosa, J., Forchhammer, K., Burillo, S., and Contreras, A. (2006). Interaction network in cyanobacterial nitrogen regulation: PipX, a protein that interacts in a 2-oxoglutarate dependent manner with PII and NtcA. Mol. Microbiol. 61, 457-469. doi: 10.1111/j.1365-2958.2006.05231.x

Espinosa, J., Forchhammer, K., and Contreras, A. (2007). Role of the Synechococcus PCC 7942 nitrogen regulator protein PipX in NtcA-controlled processes. Microbiology 153, 711-718. doi: 10.1099/mic.0.2006/003574-0

Espinosa, J., Rodriguez-Mateos, F., Salinas, P., Lanza, V. F., Dixon, R., De La Cruz, F., et al. (2014). PipX, the coactivator of NtcA, is a global regulator in cyanobacteria. Proc. Natl. Acad. Sci. U.S.A. 111, E2423-E2430. doi: 10.1073/ pnas. 1404097111

Eswaramoorthy, S., Gerchman, S., Graziano, V., Kycia, H., Studier, F. W., and Swaminathan, S. (2003). Structure of a yeast hypothetical protein selected by a structural genomics approach. Acta Crystallogr. D. Biol. Crystallogr. 59, 127-135. doi: 10.1107/S0907444902018012

Fadda, D., Pischedda, C., Caldara, F., Whalen, M. B., Anderluzzi, D., Domenici, E., et al. (2003). Characterization of divIVA and other genes located in the chromosomal region downstream of the dcw cluster in Streptococcus pneumoniae. J. Bacteriol. 185, 6209-6214. doi: 10.1128/JB.185.20.6209-6214. 2003

Feng, Z., and Barletta, R. G. (2003). Roles of Mycobacterium smegmatis $\mathrm{D}$-alanine:D-alanine ligase and $\mathrm{D}$-alanine racemase in the mechanisms of action of and resistance to the peptidoglycan inhibitor D-cycloserine. Antimicrob. Agents Chemother. 47, 283-291. doi: 10.1128/AAC.47.1.283-291. 2003

Forcada-Nadal, A., Forchhammer, K., and Rubio, V. (2014). SPR analysis of promoter binding of Synechocystis PCC6803 transcription factors NtcA and CRP suggests cross-talk and sheds light on regulation by effector molecules. FEBS Lett. 588, 2270-2276. doi: 10.1016/j.febslet.2014.05.010

Forchhammer, K. (2004). Global carbon/nitrogen control by PII signal transduction in cyanobacteria: from signals to targets. FEMS Microbiol. Rev. 28, 319-333. doi: 10.1016/j.femsre.2003.11.001

Galmozzi, C. V., Fernandez-Avila, M. J., Reyes, J. C., Florencio, F. J., and MuroPastor, M. I. (2007). The ammonium-inactivated cyanobacterial glutamine synthetase I is reactivated in vivo by a mechanism involving proteolytic removal of its inactivating factors. Mol. Microbiol. 65, 166-179. doi: 10.1111/j.1365-2958. 2007.05773.x

Garcia-Dominguez, M., Reyes, J. C., and Florencio, F. J. (2000). NtcA represses transcription of gifA and gifB, genes that encode inhibitors of glutamine synthetase type I from Synechocystis sp. PCC 6803. Mol. Microbiol. 35, 1192-1201.

Ghatge, M. S., Contestabile, R., Di Salvo, M. L., Desai, J. V., Gandhi, A. K., Camara, C. M., et al. (2012). Pyridoxal 5'-phosphate is a slow tight binding inhibitor of E. coli pyridoxal kinase. PLoS ONE 7:e41680. doi: 10.1371/journal.pone.0041680

Goclaw-Binder, H., Sendersky, E., Shimoni, E., Kiss, V., Reich, Z., Perelman, A., et al. (2012). Nutrient-associated elongation and asymmetric division of the cyanobacterium Synechococcus PCC 7942. Environ. Microbiol. 14, 680-690. doi: $10.1111 /$ j.1462-2920.2011.02620.x
Gola, S., Munder, T., Casonato, S., Manganelli, R., and Vicente, M. (2015). The essential role of SepF in mycobacterial division. Mol. Microbiol. 97, 560-576. doi: $10.1111 / \mathrm{mmi} .13050$

Golden, S. S., and Sherman, L. A. (1984). Optimal conditions for genetic transformation of the cyanobacterium Anacystis nidulans R2. J. Bacteriol. 158, $36-42$.

Guerreiro, A. C., Benevento, M., Lehmann, R., Van Breukelen, B., Post, H., Giansanti, P., et al. (2014). Daily rhythms in the cyanobacterium Synechococcus elongatus probed by high-resolution mass spectrometry-based proteomics reveals a small defined set of cyclic proteins. Mol. Cell. Proteomics 13, 2042-2055. doi: 10.1074/mcp.M113.035840

Hamoen, L. W., Meile, J. C., De Jong, W., Noirot, P., and Errington, J. (2006). SepF, a novel FtsZ-interacting protein required for a late step in cell division. Mol. Microbiol. 59, 989-999. doi: 10.1111/j.1365-2958.2005.04987.x

Hanahan, D. (1985). “Techniques for transformation of Escherichia coli”, in DNA Cloning, ed. D. Glover (Oxford: IRL Press Ltd), 109-135.

Harper, J. W., Adami, G. R., Wei, N., Keyomarsi, K., and Elledge, S. J. (1993). The p21 Cdk-interacting protein Cip1 is a potent inhibitor of G1 cyclin- dependent kinases. Cell 75, 805-816. doi: 10.1016/0092-8674(93)90499-G

Holtman, C. K., Chen, Y., Sandoval, P., Gonzales, A., Nalty, M. S., Thomas, T. L., et al. (2005). High-throughput functional analysis of the Synechococcus elongatus PCC 7942 genome. DNA Res. 12, 103-115. doi: 10.1093/dnares/12. 2.103

Huergo, L. F., and Dixon, R. (2015). The emergence of 2-oxoglutarate as a master regulator metabolite. Microbiol. Mol. Biol. Rev. 79, 419-435. doi: 10.1128/ MMBR.00038-15

Ito, T., Iimori, J., Takayama, S., Moriyama, A., Yamauchi, A., Hemmi, H., et al. (2013). Conserved pyridoxal protein that regulates Ile and Val metabolism. J. Bacteriol. 195, 5439-5449. doi: 10.1128/JB.00593-13

James, P., Halladay, J., and Craig, E. A. (1996). Genomic libraries and a host strain designed for highly efficient two- hybrid selection in yeast. Genetics 144, 1425-1436.

Klahn, S., Schaal, C., Georg, J., Baumgartner, D., Knippen, G., Hagemann, M., et al. (2015). The sRNA NsiR4 is involved in nitrogen assimilation control in cyanobacteria by targeting glutamine synthetase inactivating factor IF7. Proc. Natl. Acad. Sci. U.S.A. 112, E6243-E6252. doi: 10.1073/pnas.1508412112

Koch, A. L. (1999). Diffusion through agar blocks of finite dimensions: a theoretical analysis of three systems of practical significance in microbiology. Microbiology 145(Pt 3), 643-654. doi: 10.1099/13500872-145-3-643

Kolodkin-Gal, I., Romero, D., Cao, S., Clardy, J., Kolter, R., and Losick, R. (2010). D-amino acids trigger biofilm disassembly. Science 328, 627-629. doi: 10.1126/ science. 1188628

Labella, J. I., Obrebska, A., Espinosa, J., Salinas, P., Forcada-Nadal, A., Tremino, L., et al. (2016). Expanding the cyanobacterial nitrogen regulatory network: the GntR-like regulator PlmA interacts with the PII-PipX complex. Front. Microbiol. 7:1677. doi: 10.3389/fmicb.2016.01677

Lahmi, R., Sendersky, E., Perelman, A., Hagemann, M., Forchhammer, K., and Schwarz, R. (2006). Alanine dehydrogenase activity is required for adequate progression of phycobilisome degradation during nitrogen starvation in Synechococcus elongatus PCC 7942. J. Bacteriol. 188, 5258-5265. doi: 10.1128/ JB.00209-06

Laichoubi, K. B., Beez, S., Espinosa, J., Forchhammer, K., and Contreras, A. (2011). The nitrogen interaction network in Synechococcus WH5701, a cyanobacterium with two PipX and two $\mathrm{P}_{\mathrm{II}}$ )-like proteins. Microbiology 157, 1220-1228. doi: 10.1099/mic.0.047266-0

Laichoubi, K. B., Espinosa, J., Castells, M. A., and Contreras, A. (2012). Mutational analysis of the cyanobacterial nitrogen regulator PipX. PLoS ONE 7:e35845. doi: 10.1371/journal.pone.0035845

Langmead, B., and Salzberg, S. L. (2012). Fast gapped-read alignment with Bowtie 2. Nat. Methods 9, 357-359. doi: 10.1038/nmeth.1923

Laurent, S., Chen, H., Bedu, S., Ziarelli, F., Peng, L., and Zhang, C. C. (2005). Nonmetabolizable analogue of 2-oxoglutarate elicits heterocyst differentiation under repressive conditions in Anabaena sp. PCC 7120. Proc. Natl. Acad. Sci. U.S.A. 102, 9907-9912. doi: 10.1073/pnas.0502337102

Llacer, J. L., Contreras, A., Forchhammer, K., Marco-Marin, C., Gil-Ortiz, F., Maldonado, R., et al. (2007). The crystal structure of the complex of PII and acetylglutamate kinase reveals how PII controls the storage of nitrogen as 
arginine. Proc. Natl. Acad. Sci. U.S.A. 104, 17644-17649. doi: 10.1073/pnas. 0705987104

Llacer, J. L., Espinosa, J., Castells, M. A., Contreras, A., Forchhammer, K., and Rubio, V. (2010). Structural basis for the regulation of NtcA-dependent transcription by proteins PipX and PII. Proc. Natl. Acad. Sci. U.S.A. 107, 15397-15402. doi: 10.1073/pnas.1007015107

Lopez-Redondo, M. L., Moronta, F., Salinas, P., Espinosa, J., Cantos, R., Dixon, R., et al. (2010). Environmental control of phosphorylation pathways in a branched two-component system. Mol. Microbiol. 78, 475-489. doi: 10.1111/j.1365-2958. 2010.07348.x

Martinez, J. L., and Rojo, F. (2011). Metabolic regulation of antibiotic resistance. FEMS Microbiol. Rev. 35, 768-789. doi: 10.1111/j.1574-6976.2011.00282.x

Memon, D., Singh, A. K., Pakrasi, H. B., and Wangikar, P. P. (2013). A global analysis of adaptive evolution of operons in cyanobacteria. Antonie Van Leeuwenhoek 103, 331-346. doi: 10.1007/s10482-012-9813-0

Millson, S. H., Truman, A. W., and Piper, P. W. (2003). Vectors for N- or C-terminal positioning of the yeast Gal4p DNA binding or activator domains. Biotechniques 35, 60-64.

Miyagishima, S. Y., Wolk, C. P., and Osteryoung, K. W. (2005). Identification of cyanobacterial cell division genes by comparative and mutational analyses. Mol. Microbiol. 56, 126-143. doi: 10.1111/j.1365-2958.2005.04548.x

Moccand, C., Kaufmann, M., and Fitzpatrick, T. B. (2011). It takes two to tango: defining an essential second active site in pyridoxal 5'-phosphate synthase. PLoS ONE 6:e16042. doi: 10.1371/journal.pone.0016042

Montesinos, M. L., Herrero, A., and Flores, E. (1997). Amino acid transport in taxonomically diverse cyanobacteria and identification of two genes encoding elements of a neutral amino acid permease putatively involved in recapture of leaked hydrophobic amino acids. J. Bacteriol. 179, 853-862. doi: 10.1128/jb.179. 3.853-862.1997

Moronta-Barrios, F., Espinosa, J., and Contreras, A. (2013). Negative control of cell size in the cyanobacterium Synechococcus elongatus PCC 7942 by the essential response regulator RpaB. FEBS Lett. 587, 504-509. doi: 10.1016/j.febslet.2013. 01.023

Muro-Pastor, M. I., Reyes, J. C., and Florencio, F. J. (2001). Cyanobacteria perceive nitrogen status by sensing intracellular 2-oxoglutarate levels. J. Biol. Chem. 276, 38320-38328.

Muro-Pastor, M. I., Reyes, J. C., and Florencio, F. J. (2005). Ammonium assimilation in cyanobacteria. Photosynth. Res. 83, 135-150. doi: 10.1007/ s11120-004-2082-7

Nichols, R. J., Sen, S., Choo, Y. J., Beltrao, P., Zietek, M., Chaba, R., et al. (2011). Phenotypic landscape of a bacterial cell. Cell 144, 143-156. doi: 10.1016/j.cell. 2010.11.052

Paradis, E., Claude, J., and Strimmer, K. (2004). APE: analyses of phylogenetics and evolution in R language. Bioinformatics 20, 289-290. doi: 10.1093/ bioinformatics/btg 412

Percudani, R., and Peracchi, A. (2009). The B6 database: a tool for the description and classification of vitamin B6-dependent enzymatic activities and of the corresponding protein families. BMC Bioinformatics 10:273. doi: 10.1186/14712105-10-273

Plecko, B., Zweier, M., Begemann, A., Mathis, D., Schmitt, B., Striano, P., et al. (2017). Confirmation of mutations in PROSC as a novel cause of vitamin B6dependent epilepsy. J. Med. Genet. doi: 10.1136/jmedgenet-2017-104521 [Epub ahead of print].
Prosser, G. A., and de Carvalho, L. P. (2013). Reinterpreting the mechanism of inhibition of Mycobacterium tuberculosis D-alanine:D-alanine ligase by D-cycloserine. Biochemistry 52, 7145-7149. doi: 10.1021/bi40 $0839 f$

Prunetti, L., El Yacoubi, B., Schiavon, C. R., Kirkpatrick, E., Huang, L., Bailly, M., et al. (2016). Evidence that COG0325 proteins are involved in PLP homeostasis. Microbiology doi: 10.1099/mic.0.000255 [Epub ahead of print].

R Development Core Team (2010). R: A Language and Environment for Statistical Computing. Vienna: R Foundation for Statistical Computing.

Roder, K. H., Wolf, S. S., and Schweizer, M. (1996). Refinement of vectors for use in the yeast two-hybrid system. Anal. Biochem. 241, 260-262. doi: 10.1006/abio. 1996.0408

Rubin, B. E., Wetmore, K. M., Price, M. N., Diamond, S., Shultzaberger, R. K., Lowe, L. C., et al. (2015). The essential gene set of a photosynthetic organism. Proc. Natl. Acad. Sci. U.S.A. 112, E6634-E6643. doi: 10.1073/pnas.15192 20112

Sambrook, J., Fritsch, E., and Maniatis, T. (1989). Molecular Cloning: A Laboratory Manual. Cold Spring Harbor, NY: Cold Spring Harbor Laboratory Press.

Tanigawa, R., Shirokane, M., Maeda Si, S., Omata, T., Tanaka, K., and Takahashi, H. (2002). Transcriptional activation of NtcA-dependent promoters of Synechococcus sp. PCC 7942 by 2-oxoglutarate in vitro. Proc. Natl. Acad. Sci. U.S.A. 99, 4251-4255. doi: 10.1073/pnas.072587199

Vazquez-Bermudez, M. F., Herrero, A., and Flores, E. (2002). 2-Oxoglutarate increases the binding affinity of the NtcA (nitrogen control) transcription factor for the Synechococcus glnA promoter. FEBS Lett. 512, 71-74. doi: 10.1016/ S0014-5793(02)02219-6

Vijayan, V., Jain, I. H., and O'shea, E. K. (2011). A high resolution map of a cyanobacterial transcriptome. Genome Biol. 12:R47. doi: 10.1186/gb-2011-12$5-\mathrm{r} 47$

Wargel, R. J., Shadur, C. A., and Neuhaus, F. C. (1970). Mechanism of $\mathrm{D}$-cycloserine action: transport systems for $\mathrm{D}$-alanine, $\mathrm{D}$-cycloserine, $\mathrm{L}$-alanine, and glycine. J. Bacteriol. 103, 778-788.

Whitchurch, C. B., Hobbs, M., Livingston, S. P., Krishnapillai, V., and Mattick, J. S. (1991). Characterisation of a Pseudomonas aeruginosa twitching motility gene and evidence for a specialised protein export system widespread in eubacteria. Gene 101, 33-44. doi: 10.1016/0378-1119(91)90221-V

Zhao, M. X., Jiang, Y. L., Xu, B. Y., Chen, Y., Zhang, C. C., and Zhou, C. Z. (2010). Crystal structure of the cyanobacterial signal transduction protein PII in complex with PipX. J. Mol. Biol. 402, 552-559. doi: 10.1016/j.jmb.2010. 08.006

Conflict of Interest Statement: The authors declare that the research was conducted in the absence of any commercial or financial relationships that could be construed as a potential conflict of interest.

Copyright (C) 2017 Labella, Cantos, Espinosa, Forcada-Nadal, Rubio and Contreras. This is an open-access article distributed under the terms of the Creative Commons Attribution License (CC BY). The use, distribution or reproduction in other forums is permitted, provided the original author(s) or licensor are credited and that the original publication in this journal is cited, in accordance with accepted academic practice. No use, distribution or reproduction is permitted which does not comply with these terms. 